\title{
Direct Access to Heterocyclic Scaffolds by New Multicomponent Ugi-Smiles Couplings.
}

Laurent El Kaim*, Marie Gizolme, Laurence Grimaud*, Julie Oble.

General procedure for amino pyrimidine or amino pyridine derivative synthesis: To a $1 \mathrm{M}$ solution of carbonyl compound in methanol or toluene (see table 1 footnotes) were added the amine (1.0 equiv), the isocyanide (1.0 equiv) and the hydroxyl pyrimidine or hydroxy pyridine (1.0 equiv). The resulting mixture was stirred at $60{ }^{\circ} \mathrm{C}$ or $80{ }^{\circ} \mathrm{C}$ under an inert atmosphere and concentrated in vacuo. The crude product was then purified by flash chromatography on silica gel to give the desired adduct.

General procedure with 2-mercapto pyrimidine: To the aldehyde were added the amine (1.0 equiv), the isocyanide (1.0 equiv) and the 2-mercapto pyrimidine (1.0 equiv). The resulting mixture was stirred at $80{ }^{\circ} \mathrm{C}$ under an inert atmosphere for $12 \mathrm{~h}$ and concentrated in vacuo. The crude product was then purified by flash chromatography on silica gel to give the desired adduct.

2-( $N$-allyl- $N$-(5-nitropyridin-2-yl)amino)- $N$-tert-butylbutanamide.

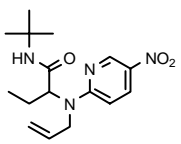

M. W. $=320.18 \mathrm{~g}^{\mathrm{mol}} \mathrm{I}^{-1}$

Yield $96 \%\left(60^{\circ} \mathrm{C}, 72 \mathrm{~h}\right)$.

${ }^{1} \mathbf{H}$ NMR $\left(\mathbf{C D C l}_{3}, 400 \mathrm{MHz}\right) \delta 9.08(\mathrm{~d}, 1 \mathrm{H}, J=2.5 \mathrm{~Hz}), 8.24(\mathrm{dd}, 1 \mathrm{H}, J=9.3,2.5 \mathrm{~Hz}), 6.55(\mathrm{~d}, 1 \mathrm{H}, J$ $=9.3 \mathrm{~Hz}), 6.22(\mathrm{br} \mathrm{s}, 1 \mathrm{H}), 5.81(\mathrm{ddd}, 1 \mathrm{H}, J=17.6,10.6,5.3 \mathrm{~Hz}), 5.26(\mathrm{dd}, 1 \mathrm{H}, J=10.6,1.3 \mathrm{~Hz}), 5.21$ $(\mathrm{dd}, 1 \mathrm{H}, J=17.6,1.3 \mathrm{~Hz}), 5.22-5.16(\mathrm{~m}, 1 \mathrm{H}), 4.25(\mathrm{dd}, 1 \mathrm{H}, J=17.6,3.5 \mathrm{~Hz}), 4.21(\mathrm{dd}, 1 \mathrm{H}, J=17.6$, $3.5 \mathrm{~Hz}$ ), 2.15-2.04 (m, 1H), 1.79-1.67 (m, 1H), 1.29 (s, 9H), 0.93 (t, 3H, $J=7.5 \mathrm{~Hz})$.

${ }^{13}$ C NMR (CDCl 3 , 100.6 MHz) $\delta 169.8,161.0,146.0,136.1,133.3,133.2,118.1,107.1,60.8,51.7$, $48.4,29.1,22.2,11.2$.

MS (DI, CI NH $\mathbf{N H}_{3} \mathrm{~m} / z 320$.

I.R. (thin film) 3361, 2973, 2881, 1677, 1592, 1495, 1329, 1295, $1121 \mathrm{~cm}^{-1}$.

HRMS Calcd. for $\mathrm{C}_{16} \mathrm{H}_{24} \mathrm{~N}_{4} \mathrm{O}_{3} 320.1848$, Found 320.1845 .

2-(N-(4-chlorobenzyl)- $N$-(3-nitropyridin-2-yl)amino)- $N$-cyclohexylbutanamide.

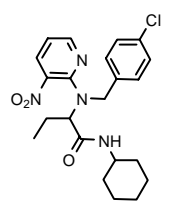

Yield $68 \%\left(60^{\circ} \mathrm{C}, 16 \mathrm{~h}\right)$.

M. W. $=430.18 \mathrm{~g}^{\mathrm{mol}} \mathrm{l}^{-1}$

${ }^{1} \mathbf{H}$ NMR $\left(\mathbf{C D C l}_{3}, 400 \mathrm{MHz}\right) \delta 8.39(\mathrm{dd}, 1 \mathrm{H}, J=4.5,1.7 \mathrm{~Hz}), 8.00(\mathrm{dd}, 1 \mathrm{H}, J=8.1,1.7 \mathrm{~Hz}), 7.32(\mathrm{br}$ $\mathrm{s}, 1 \mathrm{H}), 7.11(\mathrm{~d}, 2 \mathrm{H}, J=8.3 \mathrm{~Hz}), 6.91(\mathrm{~d}, 2 \mathrm{H}, J=8.3 \mathrm{~Hz}), 6.81(\mathrm{dd}, 1 \mathrm{H}, J=8.1,4.5 \mathrm{~Hz}), 4.79(\mathrm{dd}, 1 \mathrm{H}$, $J=8.8,6.3 \mathrm{~Hz}), 4.67(\mathrm{~d}, 1 \mathrm{H}, J=15.8 \mathrm{~Hz}), 4.55(\mathrm{~d}, 1 \mathrm{H}, J=15.8 \mathrm{~Hz}), 3.90-3.77(\mathrm{~m}, 1 \mathrm{H}), 2.18-2.00(\mathrm{~m}$, $1 \mathrm{H}), 2.06-1.96$ (m, 3H), 1.88-1.56 (m, 6H), 1.46-1.14 (m, 2H), 1.00 (t, 3H, J = 7.3 Hz).

${ }^{13} \mathbf{C}$ NMR $\left(\mathbf{C D C l}_{3}\right.$, 100.6 MHz) $\delta 170.0,152.4,151.8,135.8,135.1,134.8,133.6,129.9,128.9,115.2$, $66.1,49.7,48.4,33.5,33.4,25.9,25.0,22.9,11.3$.

MS (DI, CI NH N $_{3} \mathrm{~m} / \mathrm{z} 431$.

I.R. (thin film) 3323, 2935, 2861, 1667, 1515, 1335, 1256, $1092 \mathrm{~cm}^{-1}$. 
2-(N-(4-chlorobenzyl)- $N$-(5-(trifluoromethyl)pyridin-2-yl)amino)- $N$-cyclohexylbutanamide.

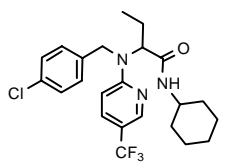

Yield $(44 \%, 72 \mathrm{~h})$.

$$
\text { M. W. }=453.18 \text { g.mol }{ }^{-1}
$$

${ }^{1} \mathbf{H}$ NMR $\left(\mathbf{C D C l}_{3}, \mathbf{4 0 0} \mathbf{M H z}\right) \delta 8.44(\mathrm{~d}, 1 \mathrm{H}, J=2.5 \mathrm{~Hz}), 7.57(\mathrm{dd}, 1 \mathrm{H}, J=8.8,2.5 \mathrm{~Hz}), 7.30(\mathrm{~d}, 2 \mathrm{H}, J$ $=8.6 \mathrm{~Hz}), 7.15(\mathrm{~d}, 2 \mathrm{H}, J=8.6 \mathrm{~Hz}), 6.42(\mathrm{~d}, 1 \mathrm{H}, J=7.8 \mathrm{~Hz}), 6.36(\mathrm{~d}, 1 \mathrm{H}, J=8.8 \mathrm{~Hz}), 5.19(\mathrm{dd}, 1 \mathrm{H}, J$ $=8.8,6.3 \mathrm{~Hz}), 4.80(\mathrm{~d}, 1 \mathrm{H}, J=17.6 \mathrm{~Hz}), 4.63(\mathrm{~d}, 1 \mathrm{H}, J=17.6 \mathrm{~Hz}), 3.78-3.67(\mathrm{~m}, 1 \mathrm{H}), 2.16-2.04(\mathrm{~m}$, $1 \mathrm{H}), 1.93-1.84(\mathrm{~m}, 1 \mathrm{H}), 1.75-1.50(\mathrm{~m}, 6 \mathrm{H}), 1.46-1.25(\mathrm{~m}, 3 \mathrm{H}), 1.22-1.09(\mathrm{~m}, 1 \mathrm{H}), 0.94(\mathrm{t}, 3 \mathrm{H}, J=7.8$ $\mathrm{Hz})$.

${ }^{13} \mathbf{C}$ NMR $\left(\mathbf{C D C l}_{3}, \mathbf{1 0 0 . 6} \mathbf{M H z}\right) \delta 170.2,160.4,145.4,136.7,135.1,133.3,129.3,128.1,123.5$, $116.4,107.8,60.4,49.2,48.3,33.3,33.1,25.8,24.9,22.5,11.5$.

MS (DI, CI NH N $_{3} \mathrm{~m} / z 453$.

I.R. (thin film) 3321, 2933, 2864, 1672, 1529, 1318, $1135 \mathrm{~cm}^{-1}$.

2-(N-allyl- $N$-(5-(trifluoromethyl)pyridin-2-yl)amino)-N-tert-butylbutanamide.

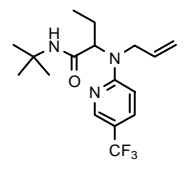

Yield $(58 \%, 16 \mathrm{~h})$.

M. W. $=343.19$ g.mol $^{-1}$

${ }^{1} \mathbf{H}$ NMR $\left(\mathbf{C D C l}_{3}, 400 \mathrm{MHz}\right) \delta 8.39(\mathrm{~d}, 1 \mathrm{H}, J=2.5 \mathrm{~Hz}), 7.64(\mathrm{dd}, 1 \mathrm{H}, J=9.1,2.5 \mathrm{~Hz}), 6.58(\mathrm{~d}, 1 \mathrm{H}, J$ $=9.1 \mathrm{~Hz}), 6.44(\mathrm{br} \mathrm{s}, 1 \mathrm{H}), 5.81(\mathrm{ddd}, 1 \mathrm{H}, J=15.6,10.1,4.8 \mathrm{~Hz}), 5.22(\mathrm{~d}, 1 \mathrm{H}, J=10.6 \mathrm{~Hz}), 5.20(\mathrm{~d}$, $1 \mathrm{H}, J=15.6 \mathrm{~Hz}), 5.13(\mathrm{t}, 1 \mathrm{H}, J=7.6 \mathrm{~Hz}), 4.15(\mathrm{dd}, 1 \mathrm{H}, J=17.4,4.8 \mathrm{~Hz}), 3.97(\mathrm{dd}, 1 \mathrm{H}, J=17.4,4.8$ $\mathrm{Hz}), 2.14-2.02(\mathrm{~m}, 1 \mathrm{H}), 1.73-1.60(\mathrm{~m}, 1 \mathrm{H}), 1.29(\mathrm{~s}, 9 \mathrm{H}), 0.92(\mathrm{t}, 3 \mathrm{H}, J=7.3 \mathrm{~Hz})$.

${ }^{13} \mathbf{C}$ NMR $\left(\mathbf{C D C l}_{3}\right.$, 100.6 MHz) $\delta 170.8,160.4,145.3,134.9,134.1,125.5,117.5,115.7,107.7,60.4$, $51.4,48.1,29.1,22.1,11.4$.

MS (DI, CI NH N $_{3} \mathrm{~m} / \mathrm{z} 344$.

I.R. (thin film) 3321, 2973, 1667, 1515, $1273 \mathrm{~cm}^{-1}$.

2-( $N$-(4-chlorobenzyl)- $N$-(2,6-dimethylpyrimidin-4-yl)amino)- $N$-cyclohexylbutanamide.

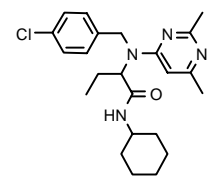

Yield $78 \%\left(60^{\circ} \mathrm{C}, 72 \mathrm{~h}\right)$.

$$
\text { M. W. }=414.22 \mathrm{~g} \cdot \mathrm{mol}^{-1}
$$

${ }^{1} \mathbf{H}$ NMR $\left(\mathrm{CDCl}_{3}, \mathbf{4 0 0} \mathbf{M H z}\right) \delta 7.28(\mathrm{~d}, 2 \mathrm{H}, J=8.3 \mathrm{~Hz}), 7.13(\mathrm{~d}, 2 \mathrm{H}, J=8.3 \mathrm{~Hz}), 6.71(\mathrm{br} \mathrm{s}, 1 \mathrm{H}), 5.95$ $(\mathrm{s}, 1 \mathrm{H}), 5.12(\mathrm{br} \mathrm{s}, 1 \mathrm{H}), 4.72(\mathrm{~d}, 1 \mathrm{H}, J=17.9 \mathrm{~Hz}), 4.52(\mathrm{~d}, 1 \mathrm{H}, J=17.9 \mathrm{~Hz}), 3.75-3.65(\mathrm{~m}, 1 \mathrm{H}), 2.56$ 
$(\mathrm{s}, 3 \mathrm{H}), 2.26(\mathrm{~s}, 3 \mathrm{H}), 2.13-1.99(\mathrm{~m}, 2 \mathrm{H}), 1.93-1.83(\mathrm{~m}, 1 \mathrm{H}), 1.73-1.49(\mathrm{~m}, 4 \mathrm{H}), 1.46-1.06(\mathrm{~m}, 5 \mathrm{H})$, $0.90(\mathrm{t}, 3 \mathrm{H}, J=7.3 \mathrm{~Hz})$.

${ }^{13}$ C NMR (CDCl 3 , 100.6 MHz) $\delta$ 170.1, 166.6, 166.3, 163.2, 136.7, 133.2, 129.3, 128.0, 100.7, 59.8, $48.6,48.1,33.4,33.1,26.6,25.9,24.9,24.8,24.7,22.3,11.4$.

MS (DI, CI NH N $_{3} \mathrm{~m} / z 415$.

I.R. (thin film) 3338, 2940, 2856, 1680, 1596, 1489, 1355, $1282 \mathrm{~cm}^{-1}$.

HRMS Calcd. for $\mathrm{C}_{23} \mathrm{H}_{31} \mathrm{ClN}_{4} 0$ 414.2186, Found 414.2179.

2-( $N$-(4-chlorobenzyl)- $N$-(2,6-dimethylpyrimidin-4-yl)amino)- $N$-cyclohexyl-2methylbutanamide.

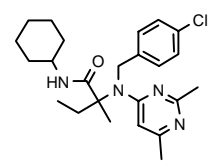

Yield $54 \%\left(60^{\circ} \mathrm{C}, 8 \mathrm{~d}\right)$.

$$
\text { M. W. }=428.23 \text { g.mol } \mathrm{l}^{-1}
$$

${ }^{1} \mathbf{H}$ NMR $\left(\mathbf{C D C l}_{\mathbf{3}}, \mathbf{4 0 0} \mathbf{M H z}\right) \delta 7.42(\mathrm{~d}, 2 \mathrm{H}, J=8.3 \mathrm{~Hz}), 7.33(\mathrm{~d}, 2 \mathrm{H}, J=8.3 \mathrm{~Hz}), 6.03(\mathrm{~s}, 1 \mathrm{H}), 5.73$ $(\mathrm{d}, 1 \mathrm{H}, J=7.8 \mathrm{~Hz}), 4.89(\mathrm{~d}, 1 \mathrm{H}, J=17.6 \mathrm{~Hz}), 4.63(\mathrm{~d}, 1 \mathrm{H}, J=17.6 \mathrm{~Hz}), 3.78-3.67(\mathrm{~m}, 1 \mathrm{H}), 2.46(\mathrm{~s}$, $3 \mathrm{H}), 2.48-2.37(\mathrm{~m}, 1 \mathrm{H}), 2.26(\mathrm{~s}, 3 \mathrm{H}), 1.90-1.73(\mathrm{~m}, 3 \mathrm{H}), 1.67-1.54(\mathrm{~m}, 3 \mathrm{H}), 1.50(\mathrm{~s}, 3 \mathrm{H}), 1.41-0.97$ (m, 5H), 0.85 (t, 3H, $J=7.5 \mathrm{~Hz})$.

${ }^{13} \mathbf{C}$ NMR $\left(\mathbf{C D C l}_{3}, \mathbf{1 0 0 . 6} \mathbf{M H z}\right) \delta 174.6,166.1,165.6,162.7,138.7,133.2,129.4,128.3,101.4,66.4$, $48.6,48.5,33.3,28.2,26.4,25.9,25.1,24.8,22.3,8.9$.

MS (DI, CI NH N $_{3} \mathrm{~m} / z 429$.

I.R. (thin film) 3361, 2940, 2856, 1663, 1584, 1495, 1295, $1091 \mathrm{~cm}^{-1}$.

HRMS Calcd. for $\mathrm{C}_{24} \mathrm{H}_{33} \mathrm{ClN}_{4} 0$ 428.2343, Found 428.2342.

2-(N-(4-chlorobenzyl)- $N$-(2-isopropyl-6-methylpyrimidin-4-yl)amino)- $N$-cyclohexyl butanamide.

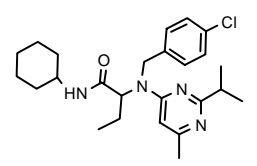

Yield $63 \%\left(60^{\circ} \mathrm{C}, 16 \mathrm{~h}\right)$.

$$
\text { M. W. }=442.25 \text { g.mol }{ }^{-1}
$$

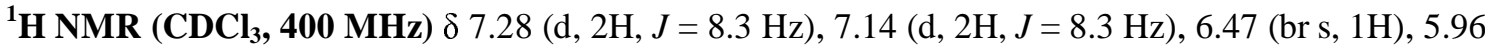
(s, 1H), $5.22(\mathrm{br} \mathrm{s}, 1 \mathrm{H}), 4.73(\mathrm{~d}, 1 \mathrm{H}, J=17.9 \mathrm{~Hz}), 4.52(\mathrm{~d}, 1 \mathrm{H}, J=17.9 \mathrm{~Hz}), 3.74-3.64(\mathrm{~m}, 1 \mathrm{H}), 3.06$ (sept., $1 \mathrm{H}, J=6.8 \mathrm{~Hz}), 2.28(\mathrm{~s}, 3 \mathrm{H}), 2.18-1.99(\mathrm{~m}, 1 \mathrm{H}), 1.95-1.85(\mathrm{~m}, 1 \mathrm{H}), 1.75-1.57(\mathrm{~m}, 5 \mathrm{H}), 1.35(\mathrm{t}$, $6 \mathrm{H}, J=6.8 \mathrm{~Hz}), 1.31-1.19(\mathrm{~m}, 3 \mathrm{H}), 1.16-1.02(\mathrm{~m}, 2 \mathrm{H}), 0.90(\mathrm{t}, 3 \mathrm{H}, J=7.3 \mathrm{~Hz})$.

${ }^{13} \mathbf{C}$ NMR $\left(\mathbf{C D C l}_{3}, \mathbf{1 0 0 . 6}\right.$ MHz) $\delta 174.1,170.3,166.4,163.3,136.9,133.2,129.2,128.1,100.9,59.6$, 48.6, 48.5, 37.9, 33.6, 33.4, 25.8, 25.3, 25.2, 24.9, 22.5, 22.4, 22.2, 11.5.

MS (DI, CI NH N $_{3} \mathrm{~m} / z 443$.

I.R. (thin film) $3398,2948,1665,1477,1273 \mathrm{~cm}^{-1}$.

2-(N-(4-chlorobenzyl)- $N$-(6-methyl-2-phenylpyrimidin-4-yl)amino)- $N$-cyclohexyl 
butanamide.

Yield $89 \%\left(60^{\circ} \mathrm{C}, 16 \mathrm{~h}\right)$.

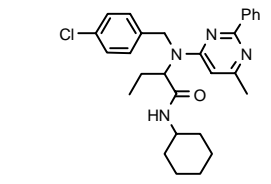

$$
\text { M. W. }=476.23 \mathrm{~g} \cdot \mathrm{mol}^{-1}
$$

${ }^{1} \mathbf{H}$ NMR $\left(\mathbf{C D C l}_{3}, \mathbf{4 0 0} \mathbf{M H z}\right) \delta 8.44-8.38(\mathrm{~m}, 2 \mathrm{H}), 7.54-7.48(\mathrm{~m}, 3 \mathrm{H}), 7.29(\mathrm{~d}, 2 \mathrm{H}, J=8.6 \mathrm{~Hz}), 7.20$ $(\mathrm{d}, 2 \mathrm{H}, J=8.6 \mathrm{~Hz}), 6.62(\mathrm{br} \mathrm{s}, 1 \mathrm{H}), 6.10(\mathrm{~s}, 1 \mathrm{H}), 5.26(\mathrm{br} \mathrm{s}, 1 \mathrm{H}), 4.76(\mathrm{~d}, 1 \mathrm{H}, J=17.2 \mathrm{~Hz}), 4.68(\mathrm{~d}$, $1 \mathrm{H}, J=17.2 \mathrm{~Hz}), 3.74-3.63(\mathrm{~m}, 1 \mathrm{H}), 2.40(\mathrm{~s}, 3 \mathrm{H}), 2.21-2.08(\mathrm{~m}, 1 \mathrm{H}), 1.84-1.66(\mathrm{~m}, 2 \mathrm{H}), 1.63-1.36(\mathrm{~m}$, $5 \mathrm{H}), 1.33-1.13(\mathrm{~m}, 4 \mathrm{H}), 0.97(\mathrm{t}, 3 \mathrm{H}, J=7.2 \mathrm{~Hz})$.

${ }^{13}$ C NMR $\left(\mathbf{C D C l}_{3}\right.$, 100.6 MHz) $\delta 170.2,166.9,163.3,138.6,138.5,136.7,133.4,130.8,129.4,128.9$, 128.3, 101.7, 59.8, 49.0, 48.1, 33.1, 32.9, 25.7, 25.0, 24.8, 24.7, 22.3, 11.6.

MS (DI, CI NH N $_{3} \mathrm{~m} / \mathrm{z} 478$.

HRMS Calcd. for $\mathrm{C}_{28} \mathrm{H}_{33} \mathrm{ClN}_{4} 0$ 476.2343, Found 476.2349.

2-(N-allyl- $N$-(6-(trifluoromethyl)-2-phenylpyrimidin-4-yl) amino)- $N$-cyclohexyl-2-

(4-methoxyphenyl)acetamide.

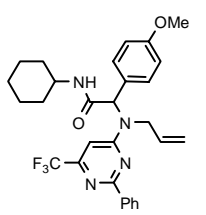

Yield $\left(51 \%, 60^{\circ} \mathrm{C}\right)$.

M. W. $=524.24$ g.mol $^{-1}$

${ }^{1} \mathbf{H}$ NMR $\left(\mathbf{C D C l}_{\mathbf{3}}, \mathbf{4 0 0} \mathbf{M H z}\right) \delta 8.43(\mathrm{dd}, 2 \mathrm{H}, J=8.1,1.8 \mathrm{~Hz}), 7.52-7.44(\mathrm{~m}, 3 \mathrm{H}), 7.37(\mathrm{~d}, 2 \mathrm{H}, J=8.6$ $\mathrm{Hz}), 6.93(\mathrm{~d}, 2 \mathrm{H}, J=8.6 \mathrm{~Hz}), 6.76(\mathrm{~s}, 1 \mathrm{H}), 6.51(\mathrm{~s}, 1 \mathrm{H}), 5.74(\mathrm{~d}, 1 \mathrm{H}, J=8.1 \mathrm{~Hz}), 5.59(\mathrm{ddd}, 1 \mathrm{H}, J=$ $15.4,10.1,5.3 \mathrm{~Hz}), 5.11(\mathrm{~d}, 1 \mathrm{H}, J=15.4 \mathrm{~Hz}), 5.11(\mathrm{~d}, 1 \mathrm{H}, J=10.1 \mathrm{~Hz}), 4.10(\mathrm{dt}, 1 \mathrm{H}, J=17.6,5.3$ $\mathrm{Hz}), 4.07(\mathrm{dt}, 1 \mathrm{H}, J=17.6,5.3 \mathrm{~Hz}), 3.91-3.81(\mathrm{~m}, 1 \mathrm{H}), 3.85(\mathrm{~s}, 3 \mathrm{H}), 1.97-1.88(\mathrm{~m}, 2 \mathrm{H}), 1.72-1.53(\mathrm{~m}$, $4 \mathrm{H}), 1.42-1.24(\mathrm{~m}, 2 \mathrm{H}), 1.20-1.03(\mathrm{~m}, 2 \mathrm{H})$.

${ }^{13} \mathbf{C}$ NMR $\left(\mathbf{C D C l}_{3}\right.$, 100.6 MHz) $\delta 169.4,164.5,163.3,160.3,155.3,137.7,133.3,131.5,131.3,128.8$, $128.7,127.2,120.2,117.8,114.7,99.3,63.4,55.7,49.0,49.1,33.4,25.8,25.2,25.1$.

MS (DI, CI NH N $_{3} m / z 525$.

HRMS Calcd. for $\mathrm{C}_{29} \mathrm{H}_{31} \mathrm{~F}_{3} \mathrm{~N}_{4} \mathrm{O}_{2}$ 524.2399, Found 524.2400.

$N$-Cyclohexyl-2-[(4-methoxy-benzyl)-(4-methyl-pyrimidin-2-yl)-amino]-butyramide.

Yield $38 \%\left(60^{\circ} \mathrm{C}, 15 \mathrm{~h}\right)$

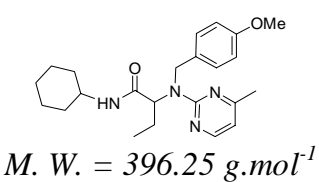

${ }^{1} \mathbf{H}$ NMR $\left(\mathbf{C D C l}_{3}, \mathbf{4 0 0} \mathbf{M H z}\right) \delta 8.23(\mathrm{~d}, 1 \mathrm{H}, J=5.3 \mathrm{~Hz}), 7.28(\mathrm{~d}, 2 \mathrm{H}, J=8.1 \mathrm{~Hz}), 6.82(\mathrm{~d}, 2 \mathrm{H}, J=8.1$ $\mathrm{Hz}), 6.47(\mathrm{~d}, 1 \mathrm{H}, J=5.3 \mathrm{~Hz}), 6.44(\mathrm{br} \mathrm{s}, 1 \mathrm{H}, \mathrm{NH}), 5.02(\mathrm{~d}, 1 \mathrm{H}, J=15.6 \mathrm{~Hz}), 4.72(\mathrm{t}, 1 \mathrm{H}, J=7.3 \mathrm{~Hz})$, $4.64(\mathrm{~d}, 1 \mathrm{H}, J=15.6 \mathrm{~Hz}), 3.79(\mathrm{~s}, 3 \mathrm{H}), 3.69-3.58(\mathrm{~m}, 1 \mathrm{H}), 2.37$ (s, 3H), 1.89-1.70 (m, 2H), 1.64-1.44 $(\mathrm{m}, 2 \mathrm{H}), 1.38-1.18(\mathrm{~m}, 4 \mathrm{H}), 1.17-0.93(\mathrm{~m}, 4 \mathrm{H}), 0.85(\mathrm{t}, 3 \mathrm{H}, J=7.3 \mathrm{~Hz})$.

${ }^{13} \mathbf{C}$ NMR $\left(\mathbf{C D C l}_{3}, \mathbf{1 0 0 . 6}\right.$ MHz) $\delta 171.2,167.7,162.3,158.9,157.6,132.3,129.5,114.1,110.5,61.9$, $55.6,48.8,47.9,33.1,32.9,25.9,24.9,24.8,22.3,11.6$. 
MS (DI, CI NH N $^{2} m / z 396$.

I.R. (thin film) 3311, 2936, 2864, 1669, 1582, 1516, 1472, 1360, $1275 \mathrm{~cm}^{-1}$.

HRMS Calcd. for $\mathrm{C}_{23} \mathrm{H}_{32} \mathrm{~N}_{4} \mathrm{O}_{2} 396.2525$, Found 396.2511.

2-[(2-Methoxy-ethyl)-pyrimidin-2-yl-amino]-4-methyl-pentanethioic acid cyclohexylamide.

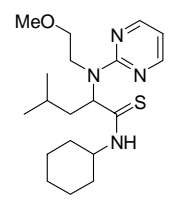

Yield $77 \%\left(80^{\circ} \mathrm{C}, 12 \mathrm{~h}\right)$

$$
\text { M. W. }=364.20 \mathrm{~g} \cdot \mathrm{mol}^{-1}
$$

${ }^{1} \mathbf{H}$ NMR (CDCl $\left.\mathbf{3}, \mathbf{4 0 0} \mathbf{M H z}\right) \delta 9.36(\mathrm{br} \mathrm{s}, 1 \mathrm{H}), 8.35(\mathrm{~d}, 2 \mathrm{H}, J=4.8 \mathrm{~Hz}), 6.61(\mathrm{t}, 1 \mathrm{H}, J=4.8 \mathrm{~Hz}), 5.21$ (br s, 1H), 4.41-4.31 (m, 1H), 3.92-3.85 (m, 1H), 3.78-3.75 (m, 2H), 3.61-3.57 (m, 1H), $3.36(\mathrm{~s}, 3 \mathrm{H})$, 2.32-2.25 (m, 1H), 2.04-1.90 (m, 3H), 1.68-1.56 (m, 2H), 1.52-1.48 (m, 1H), 1.42-1.35 (m, 2H), 1.28$1.08(\mathrm{~m}, 4 \mathrm{H}), 0.93(\mathrm{~d}, 3 \mathrm{H}, J=6.8 \mathrm{~Hz}), 0.89(\mathrm{~d}, 3 \mathrm{H}, J=6.8 \mathrm{~Hz})$.

${ }^{13} \mathbf{C}$ NMR (CDCl 3 , 100.6 MHz) $\delta$ 202.4, 162.5, 157.8, 111.4, 70.6, 59.1, 53.9, 40.7, 31.5, 31.2, 26.0, 25.5, 27.7, 24.8, 23.6, 22.4.

MS (DI, CI NH N $\mathrm{m} / \mathrm{z} 365$.

I.R. (thin film) 2929, 2854, 1585, 1507, 1477, 1359, $1116 \mathrm{~cm}^{-1}$.

HRMS Calcd. for $\mathrm{C}_{19} \mathrm{H}_{32} \mathrm{~N}_{4} \mathrm{O}_{2} \mathrm{~S} 364.2297$, Found 364.2304.

$N$-Cyclohexyl-2-cyclopropyl-2-[(4-methoxy-benzyl)-pyrimidin-2-yl-amino]-thioacetamide.

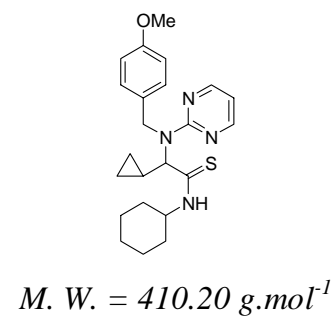

Yield $71 \%\left(80^{\circ} \mathrm{C}, 12 \mathrm{~h}\right)$

${ }^{1} \mathbf{H}$ NMR (CDCl $\left.\mathbf{3}, 400 \mathrm{MHz}\right) \delta 9.30(\mathrm{br} \mathrm{s}, 1 \mathrm{H}), 8.35$ (d, 2H, $\left.J=4.8 \mathrm{~Hz}\right), 7.30(\mathrm{~d}, 2 \mathrm{H}, J=8.8 \mathrm{~Hz}$,), $6.84(\mathrm{~d}, 2 \mathrm{H}, J=8.8 \mathrm{~Hz}), 6.61(\mathrm{t}, 1 \mathrm{H}, J=4.8 \mathrm{~Hz}), 5.22(\mathrm{~d}, 1 \mathrm{H}, J=16.0 \mathrm{~Hz}), 4.90(\mathrm{~d}, 1 \mathrm{H}, J=16.0 \mathrm{~Hz})$, 4.38-4.29 (m, 1H), $4.18(\mathrm{br} \mathrm{s}, 1 \mathrm{H}), 3.81(\mathrm{~s}, 3 \mathrm{H}), 2.01-1.95(\mathrm{~m}, 1 \mathrm{H}), 1.92-1.86(\mathrm{~m}, 1 \mathrm{H}), 1.82-1.74(\mathrm{~m}$, $1 \mathrm{H}), 1.67-1.55(\mathrm{~m}, 2 \mathrm{H}), 1.47-1.35(\mathrm{~m}, 2 \mathrm{H}), 1.28-1.14(\mathrm{~m}, 4 \mathrm{H}), 0.74-0.66(\mathrm{~m}, 1 \mathrm{H}), 0.45-0.39(\mathrm{~m}, 1 \mathrm{H})$, 0.27-0.20 (m, 1H), 0.04--0.04 (m, 1H).

${ }^{13} \mathbf{C}$ NMR $\left(\mathbf{C D C l}_{3}\right.$, 100.6 MHz) $\delta 200.8,162.4,158.9,157.9,132.0,129.3,114.0,111.2,73.0,55.7$, 53.6, 49.6, 31.5, 31.2, 25.9, 24.6, 13.3, 6.8, 5.7.

MS (DI, CI NH $\mathbf{N H}_{3} \mathrm{~m} / \mathrm{z} 410$.

I.R. (thin film) 2927, 2852, 2360, 2341, 1583, 1478, $1174 \mathrm{~cm}^{-1}$.

2-[(2-Methoxy-ethyl)-pyrimidin-2-yl-amino]-4-methyl-pentanethioicacid $\quad$ [2-(3,4-dimethoxy-phenyl)ethyl]-amide 


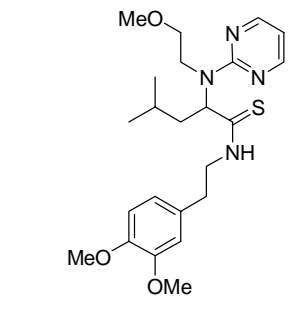

M. W. $=446.24$ g.mol ${ }^{-1}$

Yield $75 \%\left(80^{\circ} \mathrm{C}, 12 \mathrm{~h}\right)$.

${ }^{1} \mathbf{H}$ NMR $\left(\mathbf{C D C l}_{3}, 400 \mathrm{MHz}\right) \delta 9.53(\mathrm{br} \mathrm{s}, 1 \mathrm{H}), 8.22(\mathrm{~d}, 2 \mathrm{H}, J=4.8 \mathrm{~Hz}), 6.66-6.63(\mathrm{~m}, 2 \mathrm{H}), 6.60-6.55$ $(\mathrm{m}, 2 \mathrm{H}), 5.32(\mathrm{br} \mathrm{s}, 1 \mathrm{H}), 3.97-3.88(\mathrm{~m}, 4 \mathrm{H}), 3.85(\mathrm{~s}, 3 \mathrm{H}), 3.83(\mathrm{~s}, 3 \mathrm{H}), 3.77-3.71(\mathrm{~m}, 1 \mathrm{H}), 3.53-3.47$ $(\mathrm{m}, 1 \mathrm{H}), 3.28(\mathrm{~s}, 3 \mathrm{H}), 2.86(\mathrm{t}, 2 \mathrm{H}, J=6.8 \mathrm{~Hz}), 2.31-2.24(\mathrm{~m}, 1 \mathrm{H}), 2.00-1.92(\mathrm{~m}, 1 \mathrm{H}), 1.51-1.43(\mathrm{~m}$, $1 \mathrm{H}), 0.92(\mathrm{~d}, 3 \mathrm{H}, J=6.8 \mathrm{~Hz}), 0.87(\mathrm{~d}, 3 \mathrm{H}, J=6.8 \mathrm{~Hz})$.

${ }^{13} \mathbf{C ~ N M R}\left(\mathbf{C D C l}_{3}\right.$, $\left.100.6 \mathrm{MHz}\right) \delta 204.6,162.4,157.7,149.3,147.9,131.5,121.0,112.0,111.5,111.3$, $70.5,59.1,56.3,56.2,47.0,40.9,33.6,25.5,23.6,22.3$.

MS (DI, CI NH $\mathbf{N H}_{3} \mathrm{~m} / \mathrm{z} 47$.

I.R. (thin film) $2927,2360,2342,1515,1114 \mathrm{~cm}^{-1}$.

HRMS Calcd. for $\mathrm{C}_{23} \mathrm{H}_{34} \mathrm{~N}_{4} \mathrm{O}_{3} \mathrm{~S} 446.2352$, Found 446.2358.

$N$-tert-Butyl-2-[(2-methoxy-ethyl)-pyrimidin-2-yl-amino]-thiobutyramide.

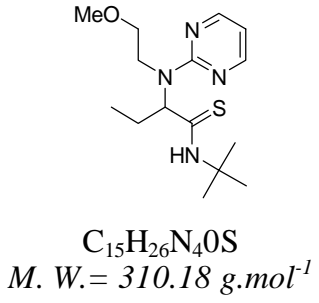

Yield $42 \%\left(80^{\circ} \mathrm{C}, 12 \mathrm{~h}\right)$.

${ }^{1} \mathbf{H}$ NMR $\left(\mathbf{C D C l}_{3}, 400 \mathrm{MHz}\right) \delta 9.52(\mathrm{br} \mathrm{s}, 1 \mathrm{H}), 8.33(\mathrm{~d}, 2 \mathrm{H}, J=4.8 \mathrm{~Hz}), 6.59(\mathrm{t}, 1 \mathrm{H}, J=4.8 \mathrm{~Hz}), 4.80$ (br s, 1H), $3.86(\mathrm{t}, 2 \mathrm{H}, J=6.0 \mathrm{~Hz}), 3.69-3.63(\mathrm{~m}, 1 \mathrm{H}), 3.62-3.56(\mathrm{~m}, 1 \mathrm{H}), 3.34(\mathrm{~s}, 3 \mathrm{H}), 2.45-2.34(\mathrm{~m}$, $1 \mathrm{H}), 2.14-2.03(\mathrm{~m}, 1 \mathrm{H}), 1.46(\mathrm{~s}, 9 \mathrm{H}), 0.87(\mathrm{t}, 3 \mathrm{H}, J=7.3 \mathrm{~Hz})$.

${ }^{13} \mathbf{C ~ N M R}\left(\mathbf{C D C l}_{3}\right.$, 100.6 MHz) $\delta 202.1,162.2,157.7,111.3,70.6,59.1,55.3,46.2,25.8,24.5,11.6$.

MS (DI, CI NH $\left.\mathbf{N H}_{3}\right) \mathrm{m} / \mathrm{z} 31\left(\mathrm{M}+\mathrm{H}^{+}\right)$.

I.R. (thin film) 2964, 2360, 2341, 1585, 1476, $1116 \mathrm{~cm}^{-1}$.

HRMS Calcd. for $\mathrm{C}_{15} \mathrm{H}_{26} \mathrm{~N}_{4} 0 \mathrm{~S} 310.1827$, Found 310.1840 


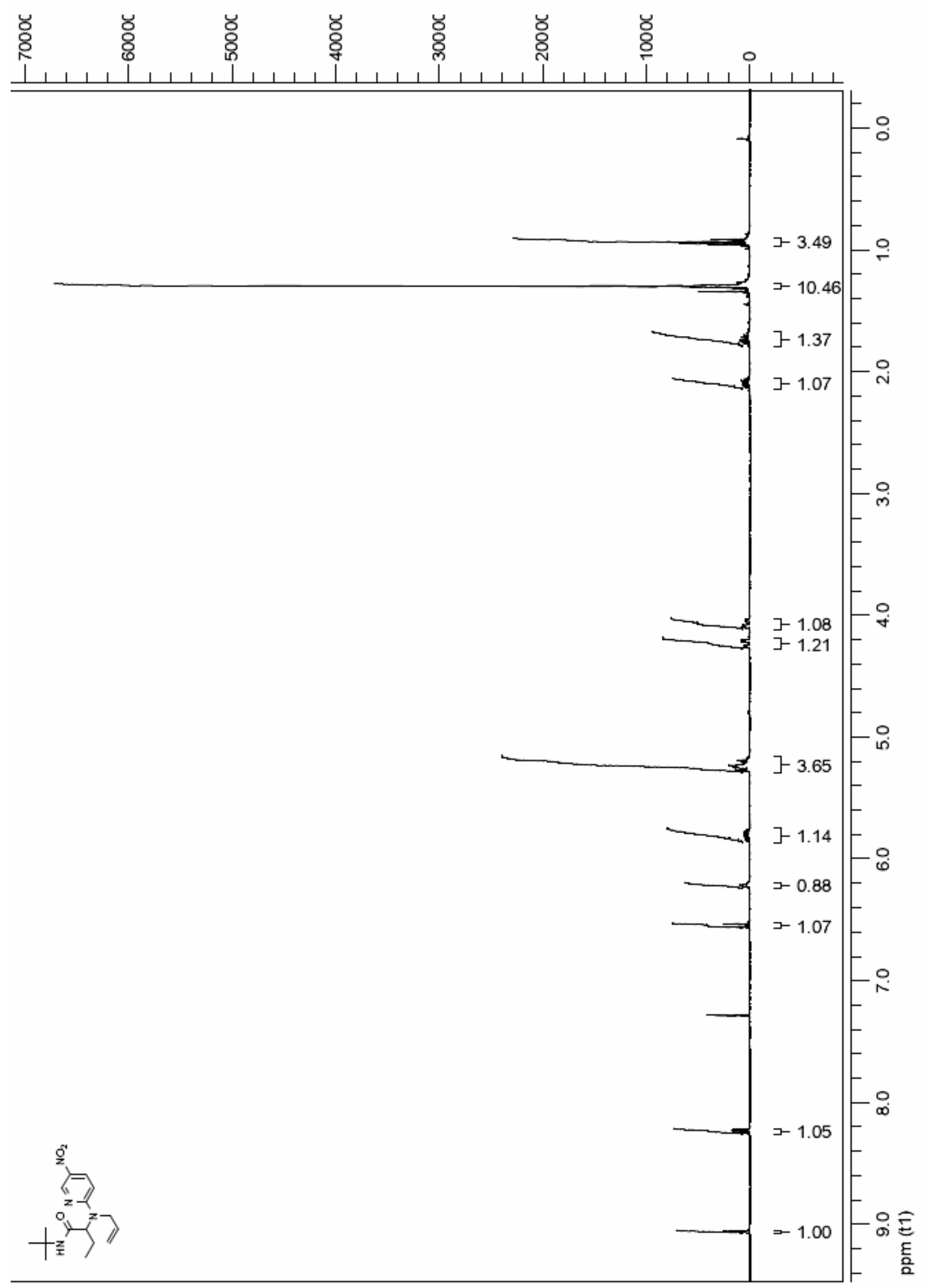

Table 1, Entry 1. 


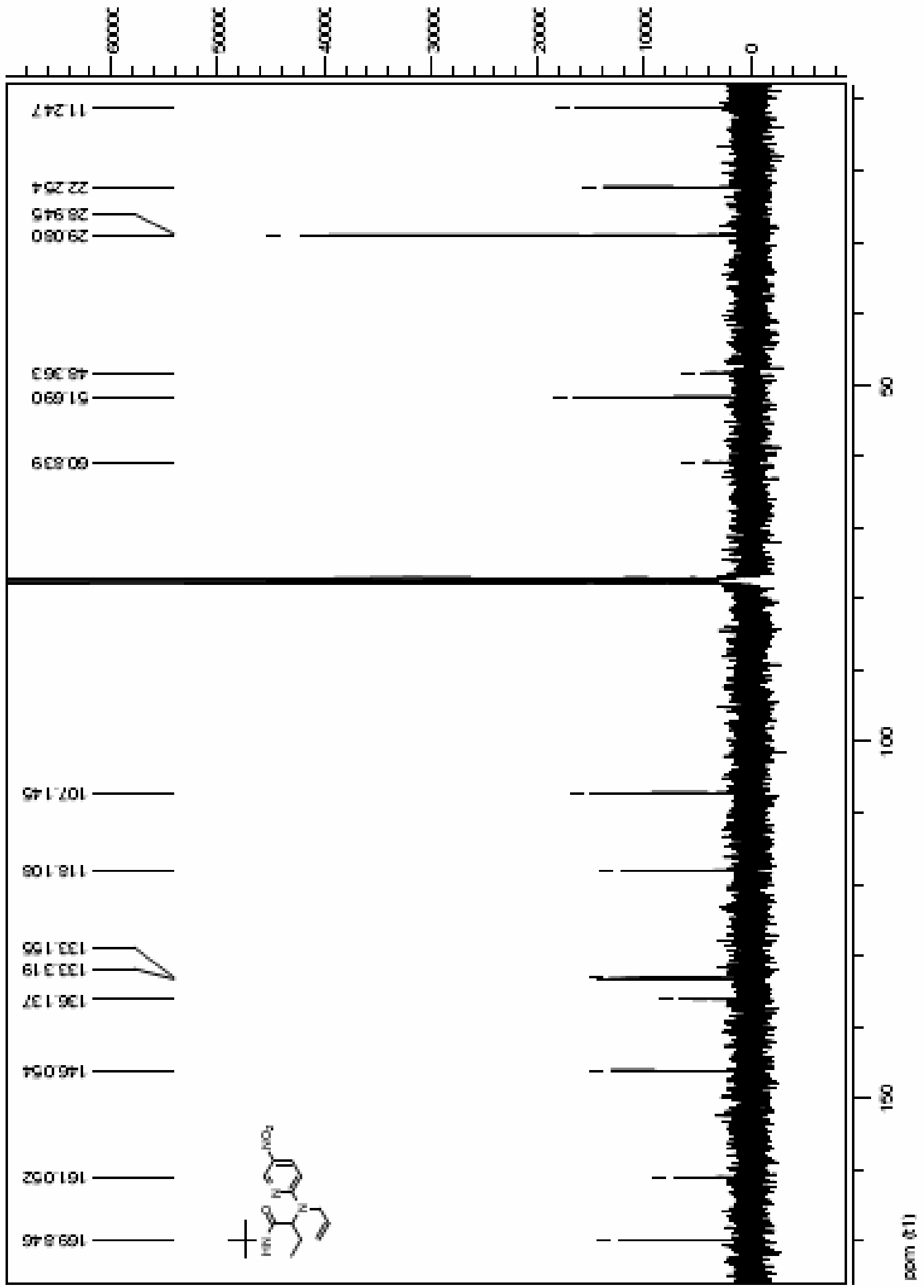

Table 1, Entry 1. 


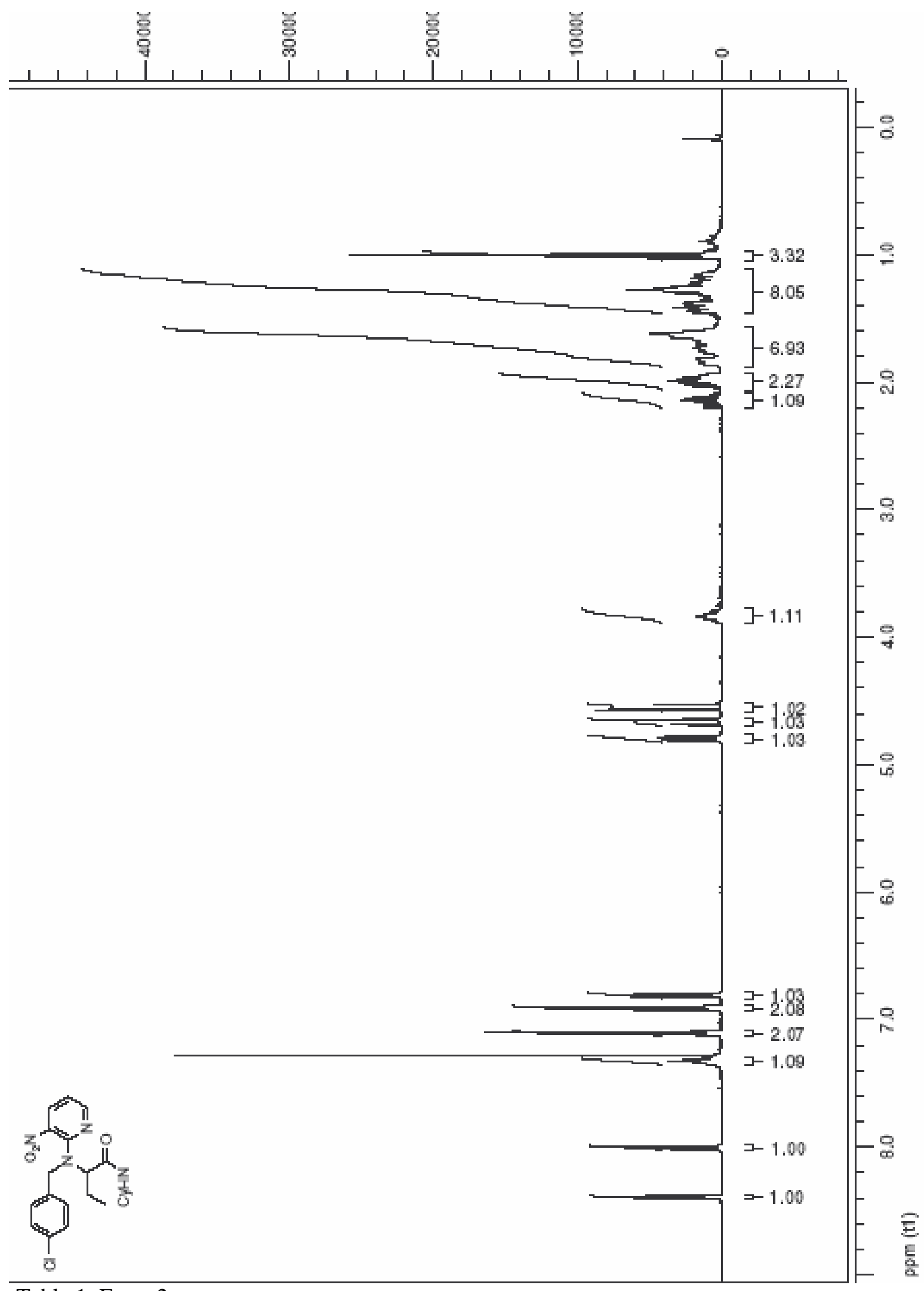

Table 1, Entry 2. 


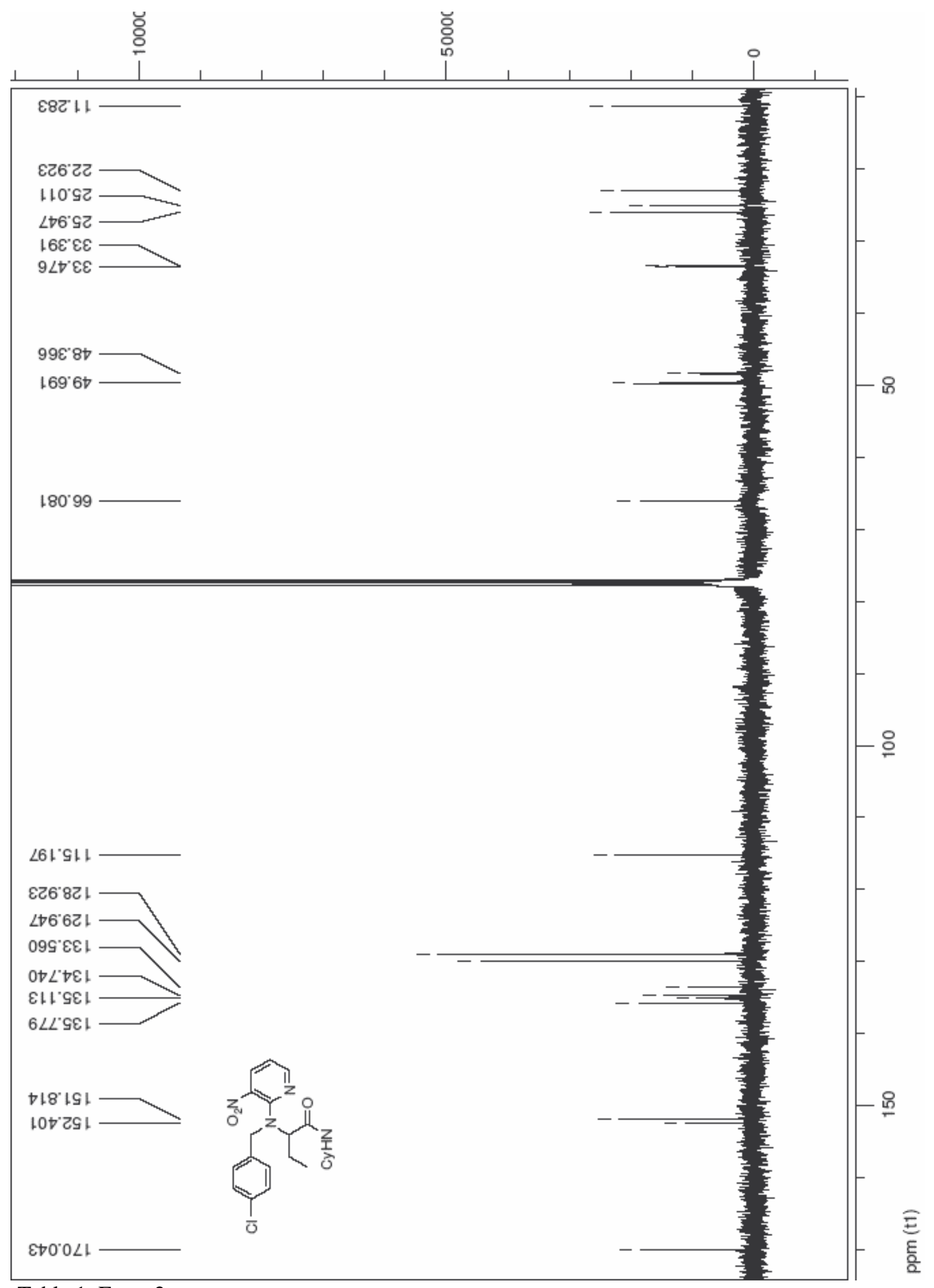

Table 1, Entry 2. 


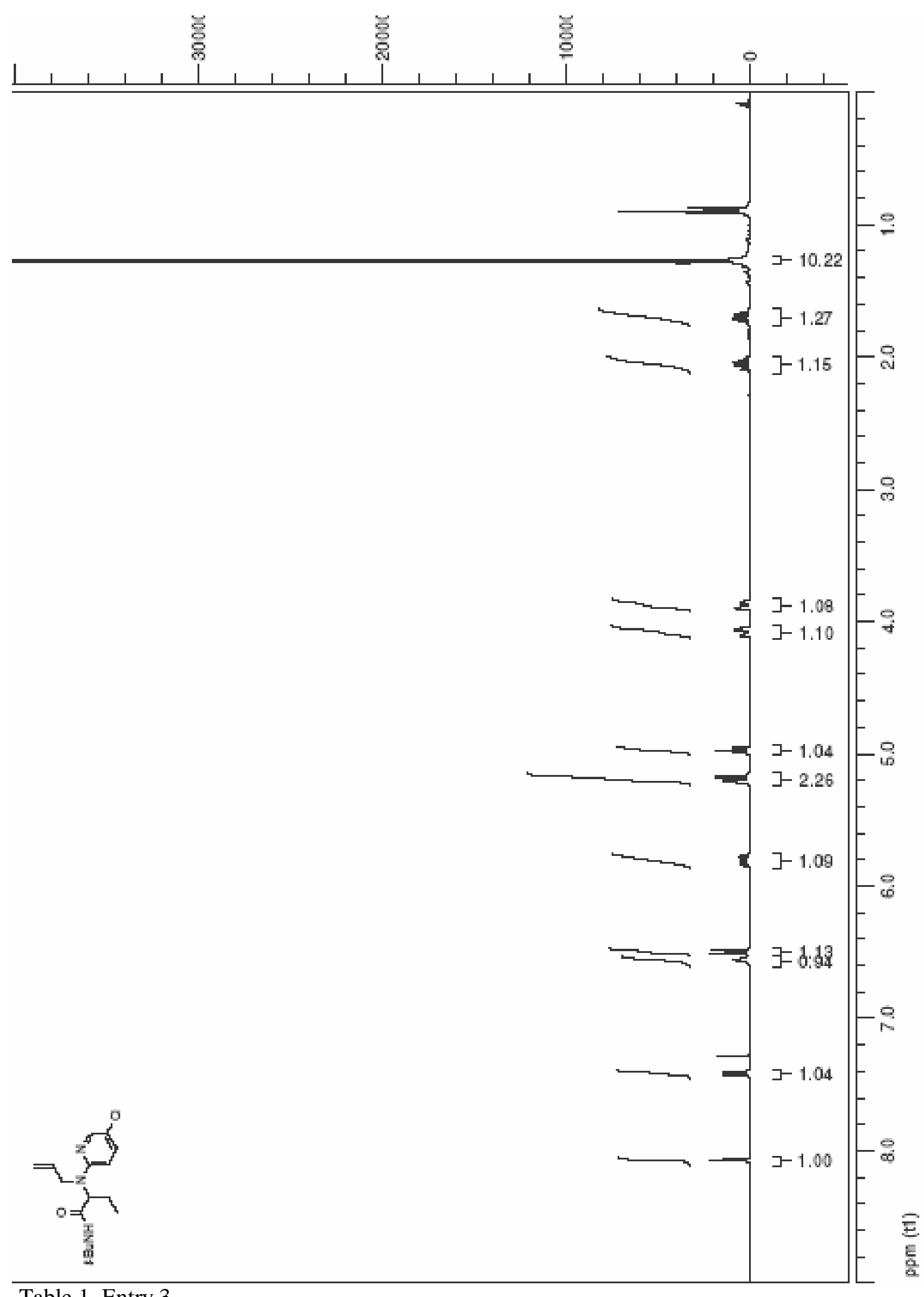

Table 1, Entry 3. 


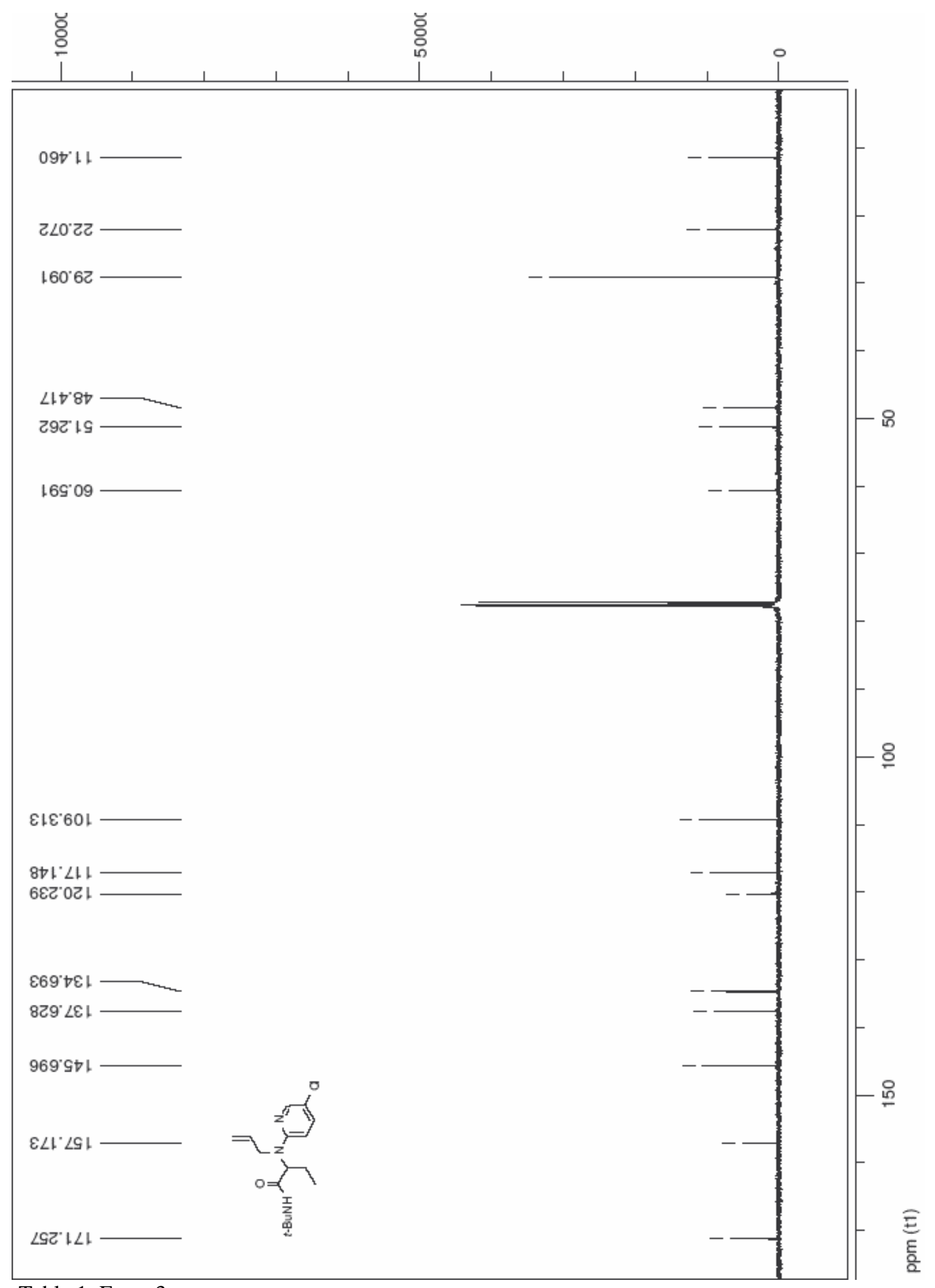

Table 1, Entry 3. 


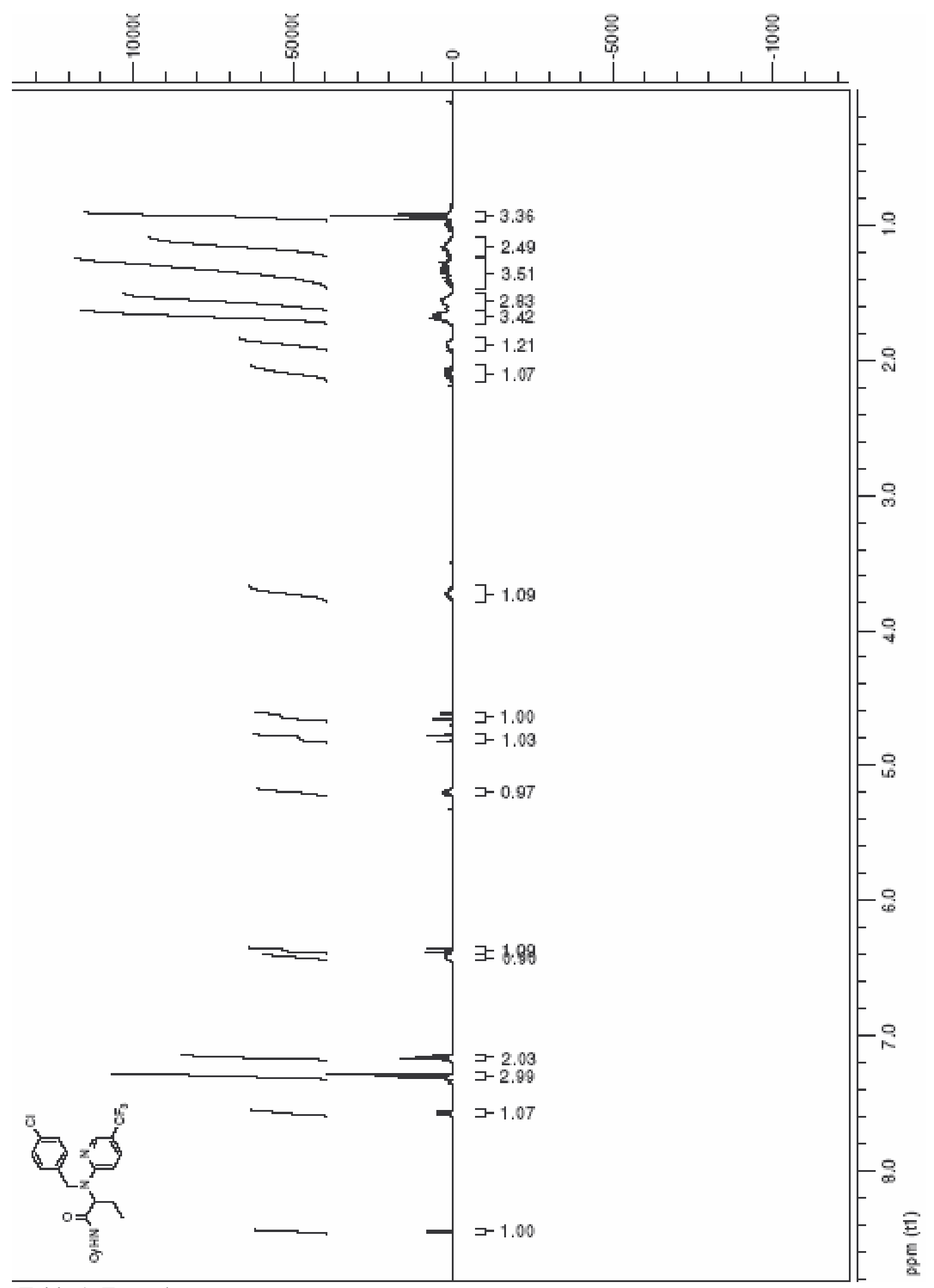

Table 1, Entry 4. 


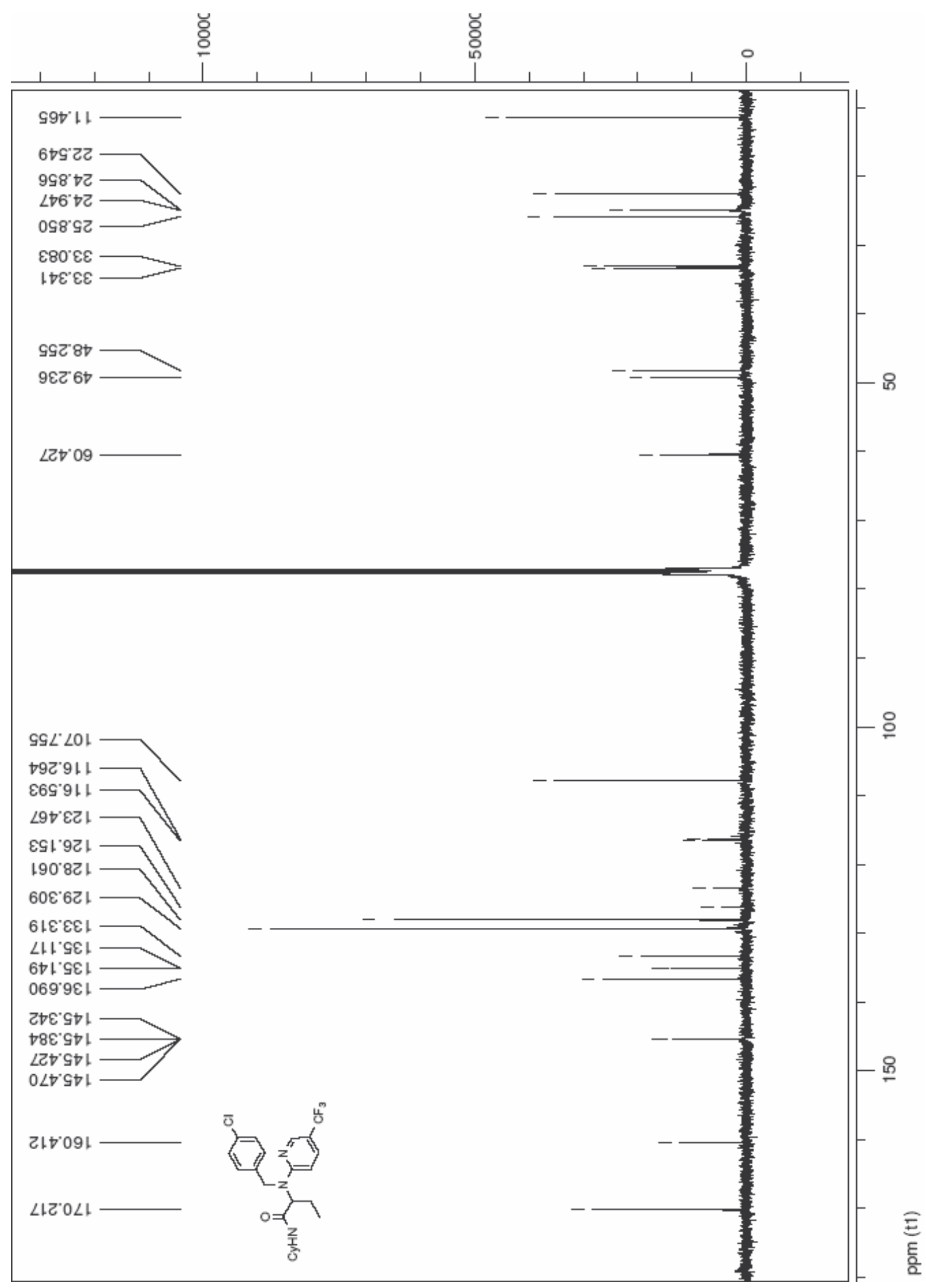

Table 1, Entry 4. 


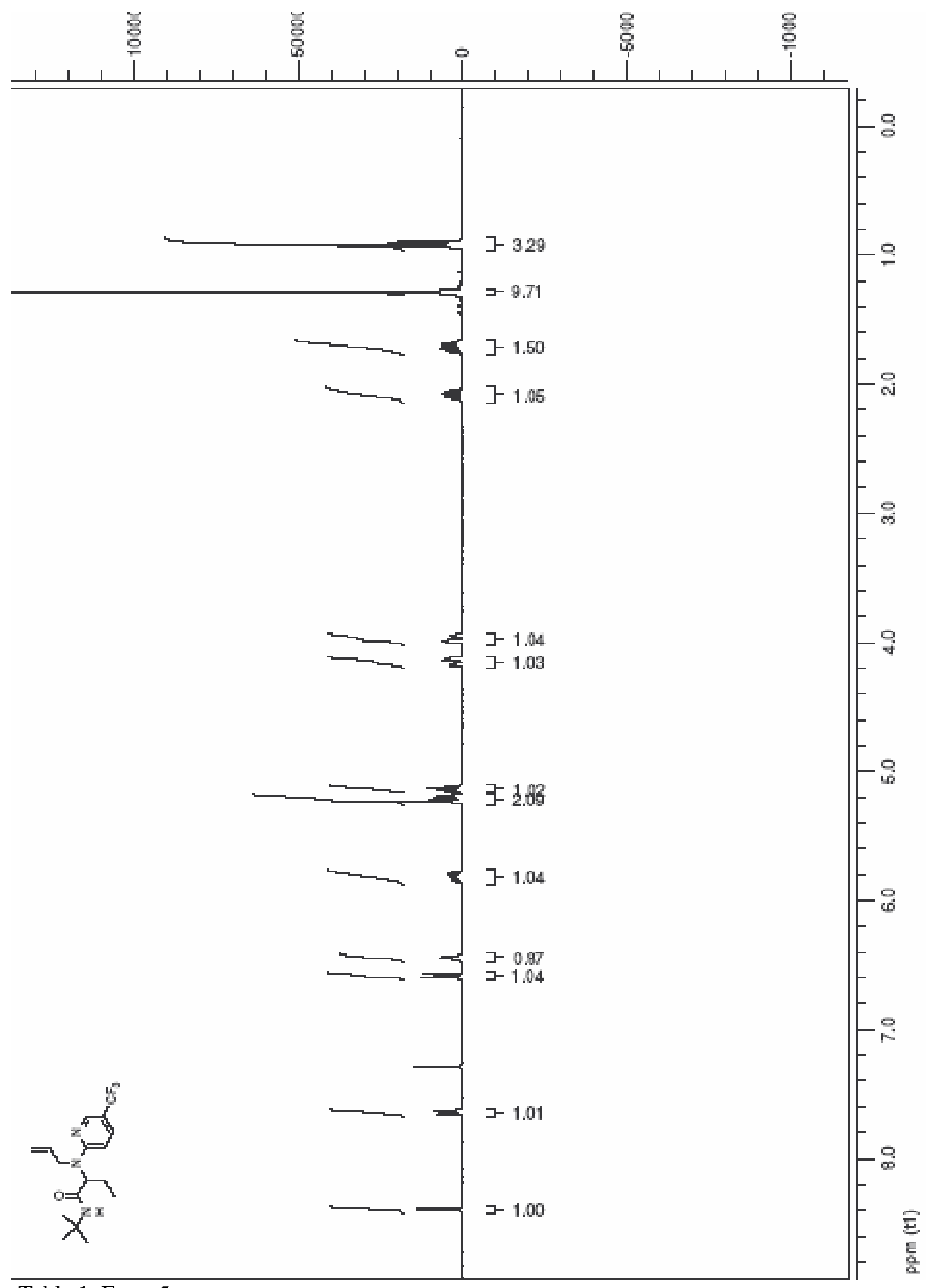

Table 1, Entry 5. 


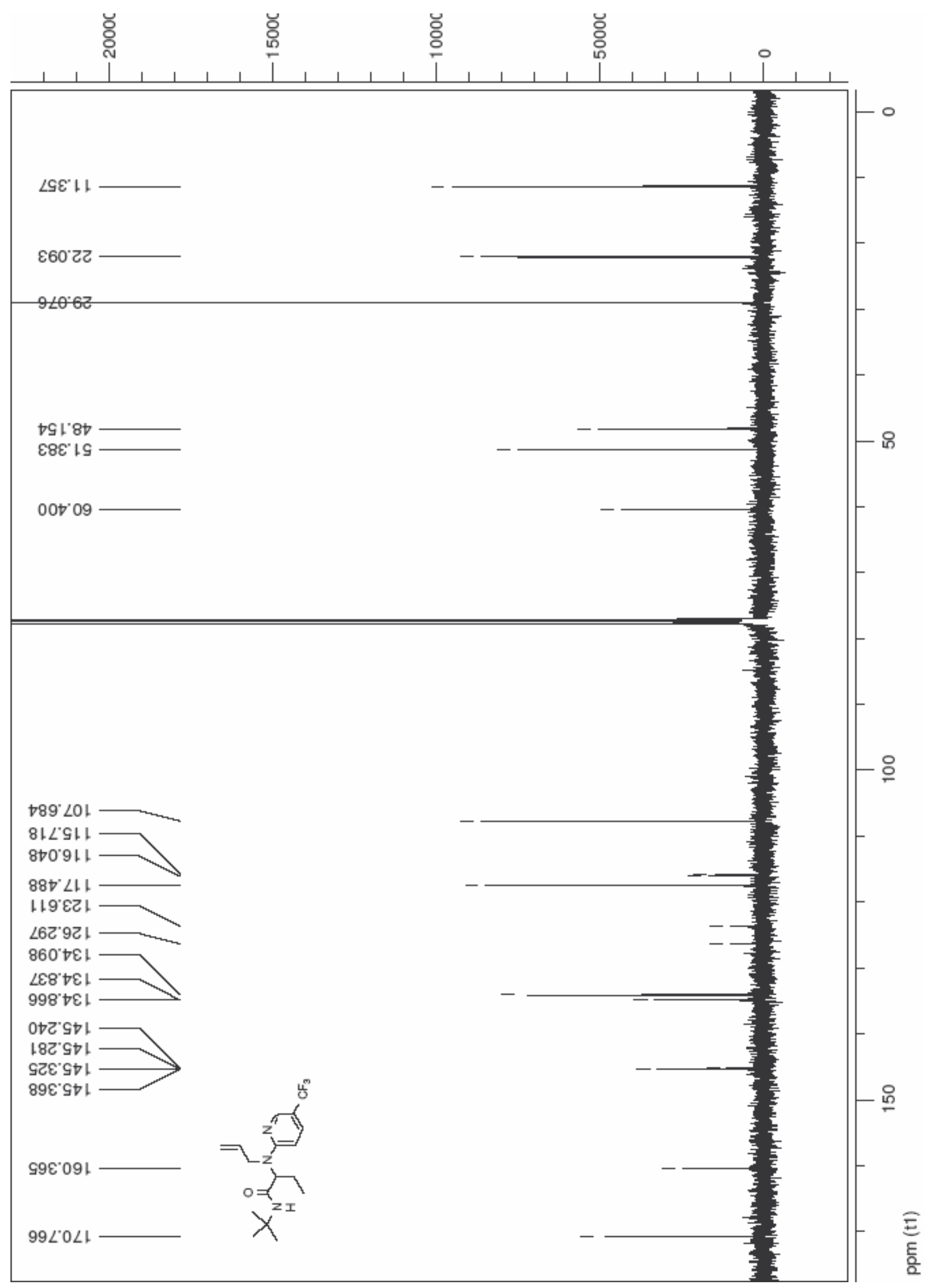

Table 1, Entry 5. 


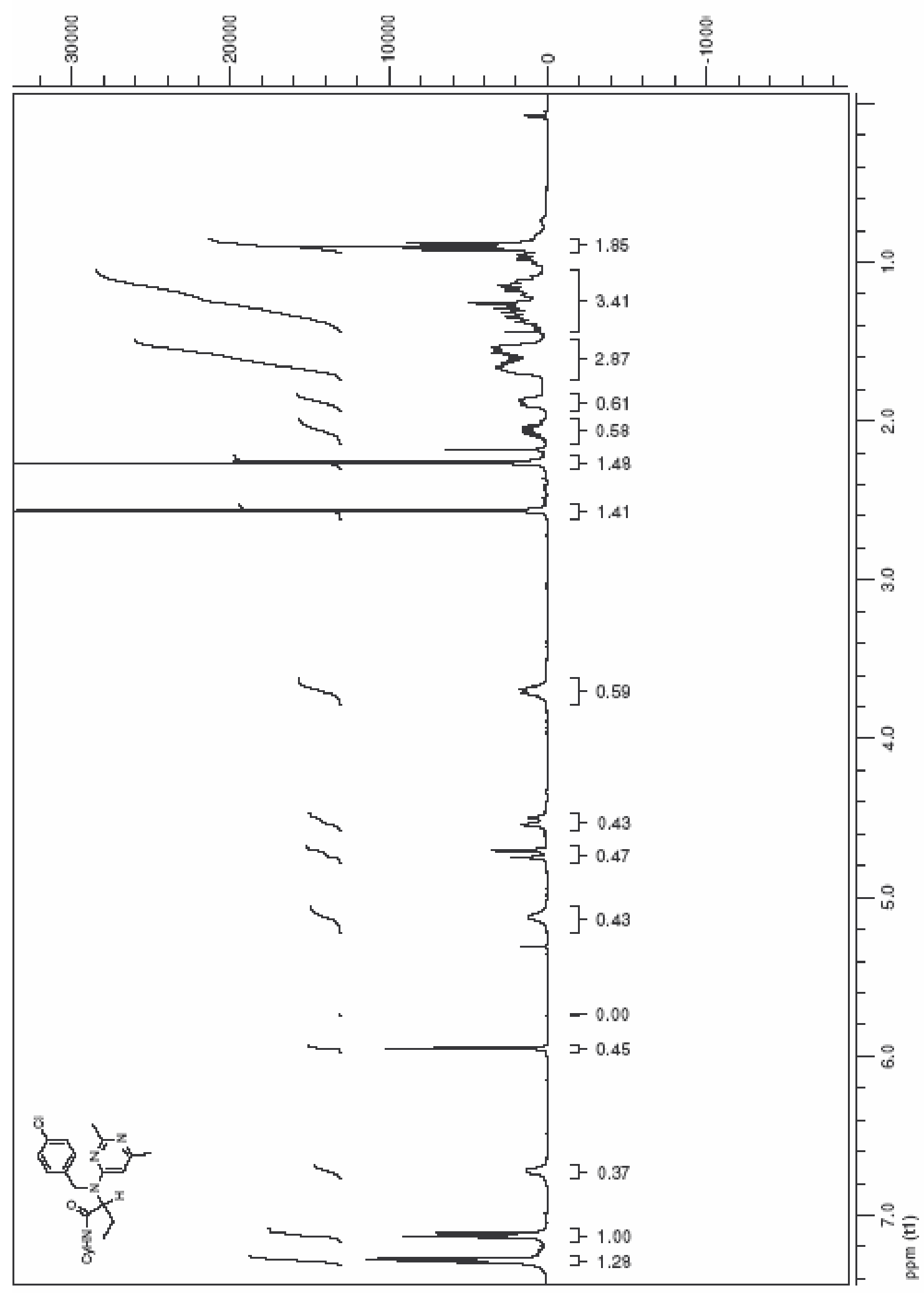

Table 1, Entry 6A. 


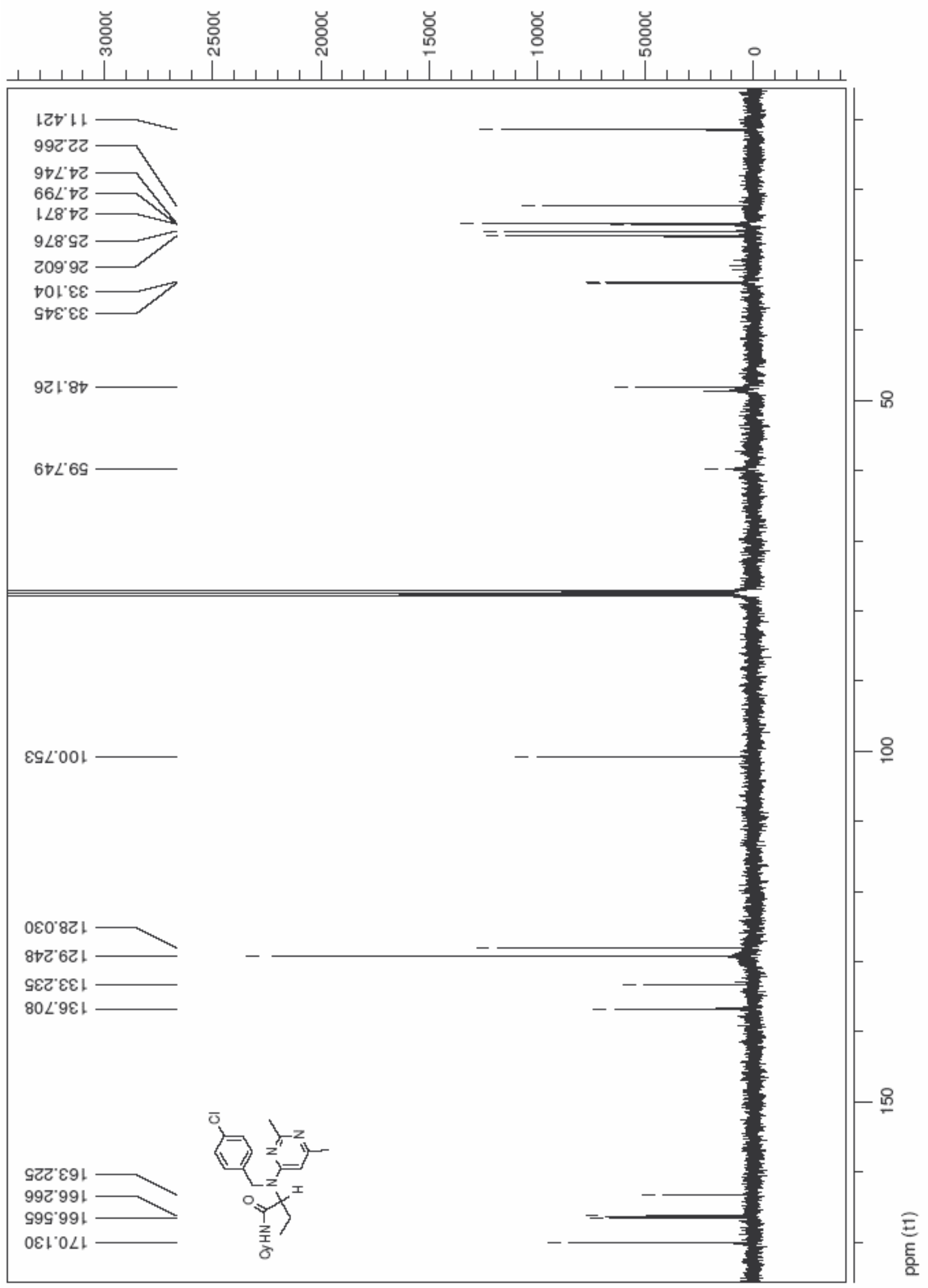

Table 1, Entry 6A. 


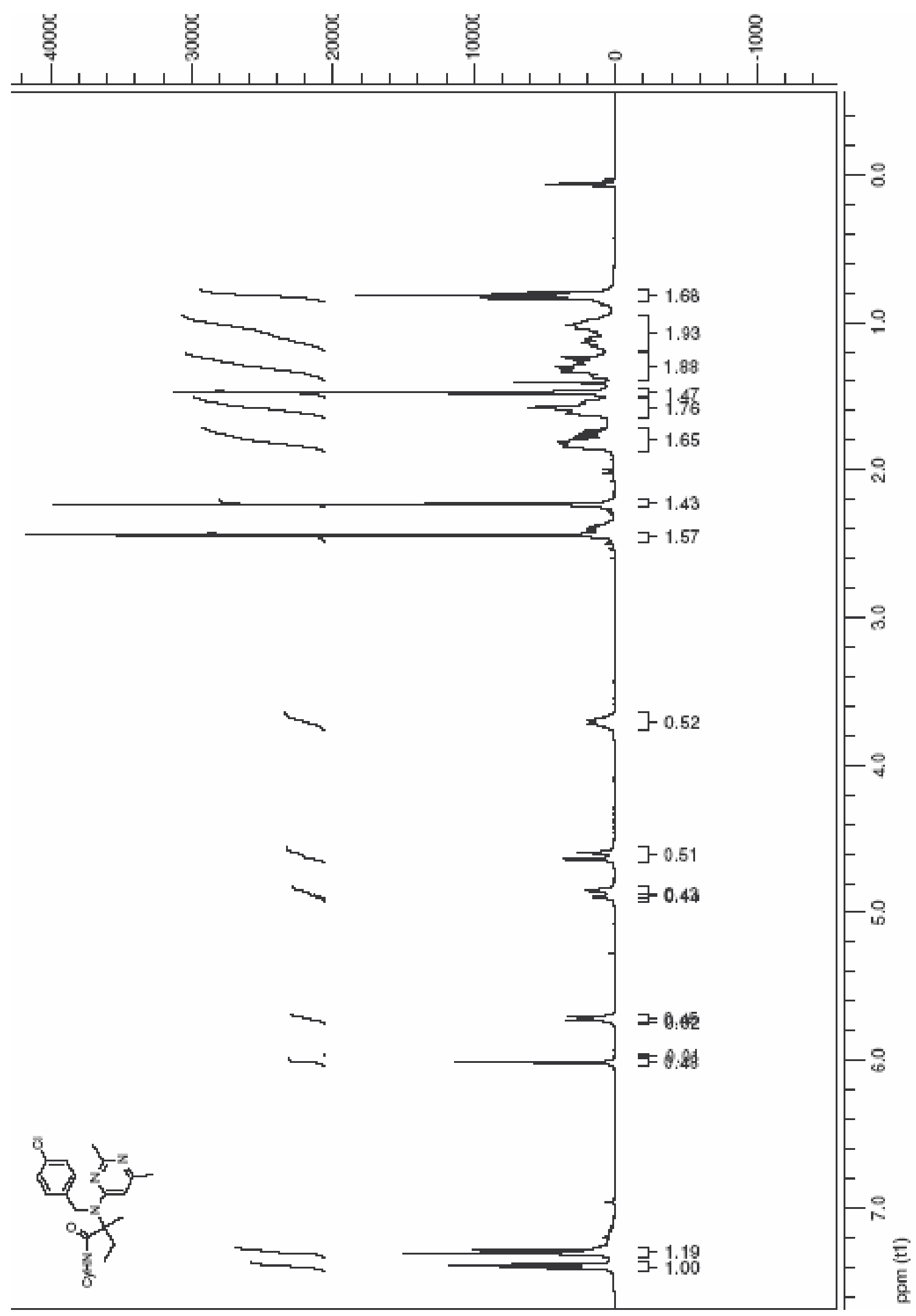

Table 1, Entry 6B. 


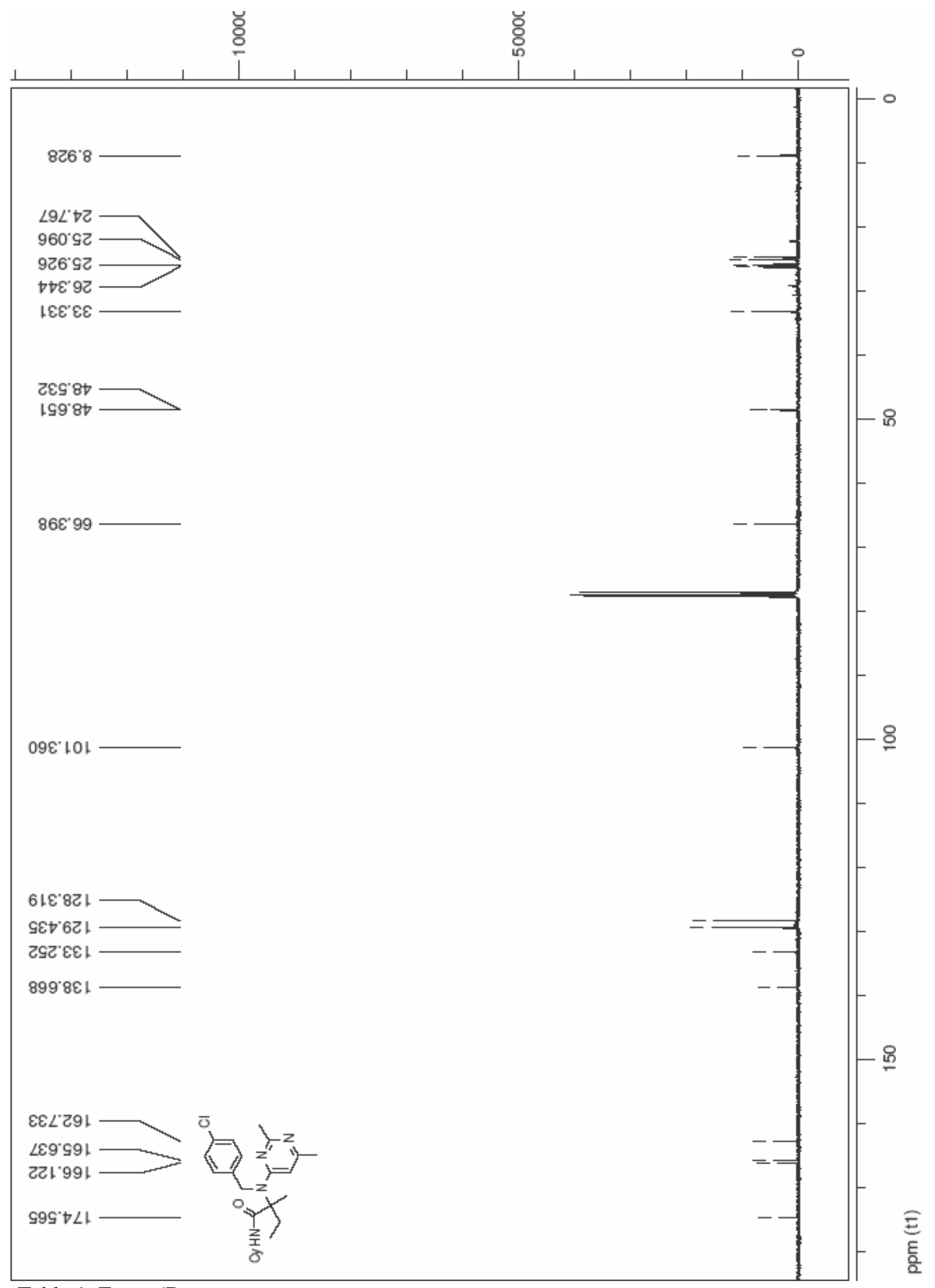

Table 1, Entry 6B. 
Table 1, Entry 7.

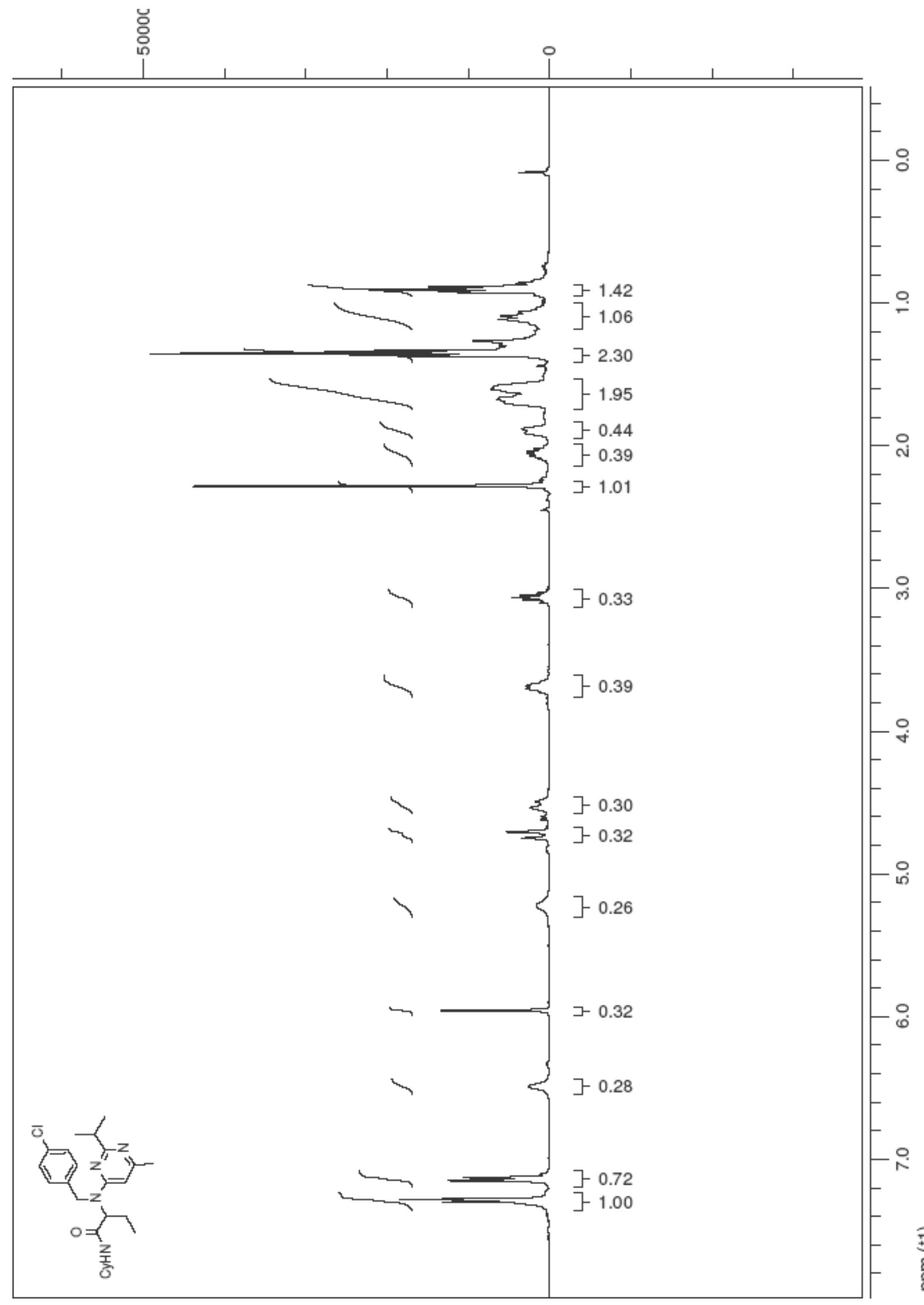

产 


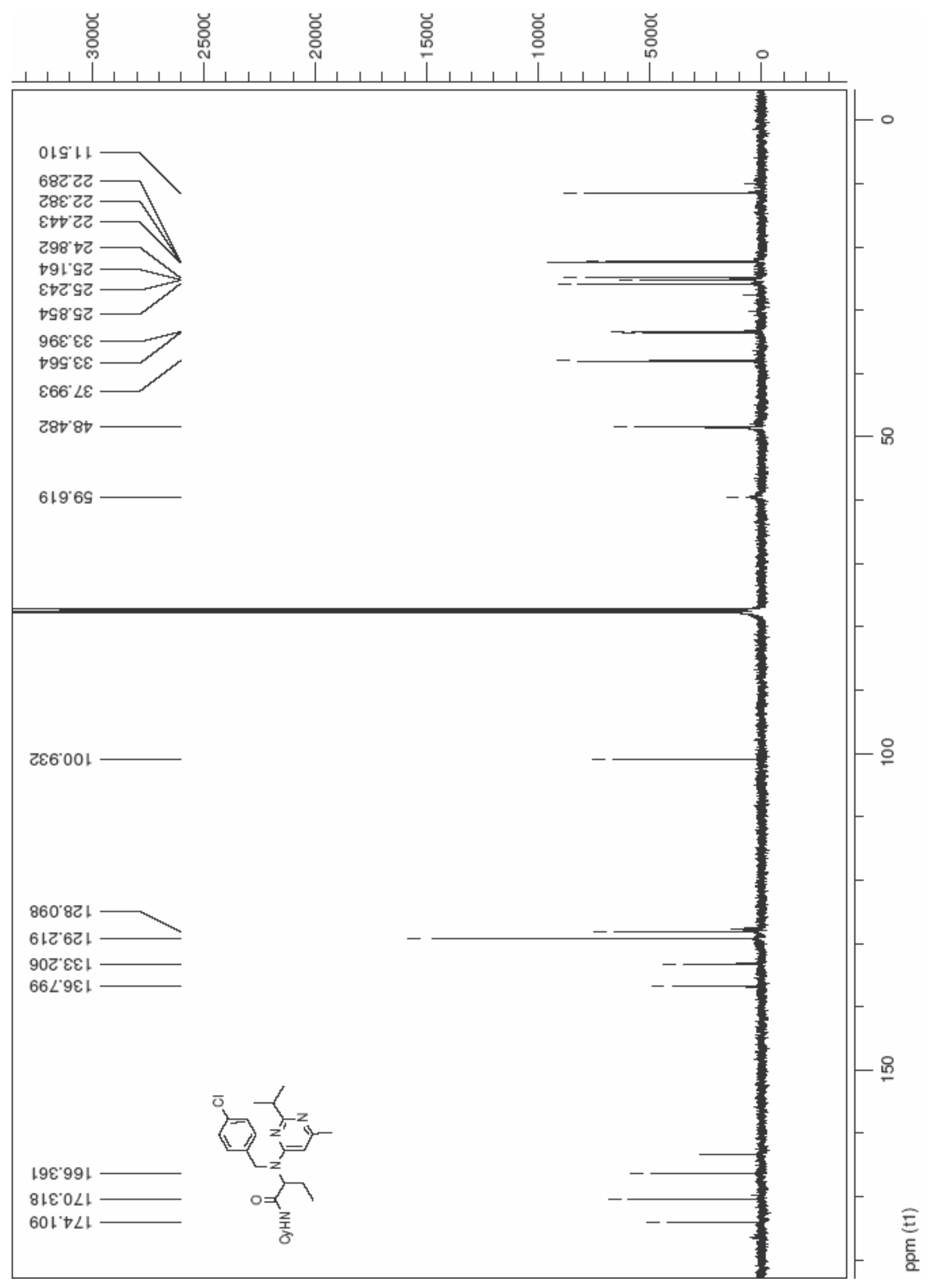


Table 1, Entry 7.

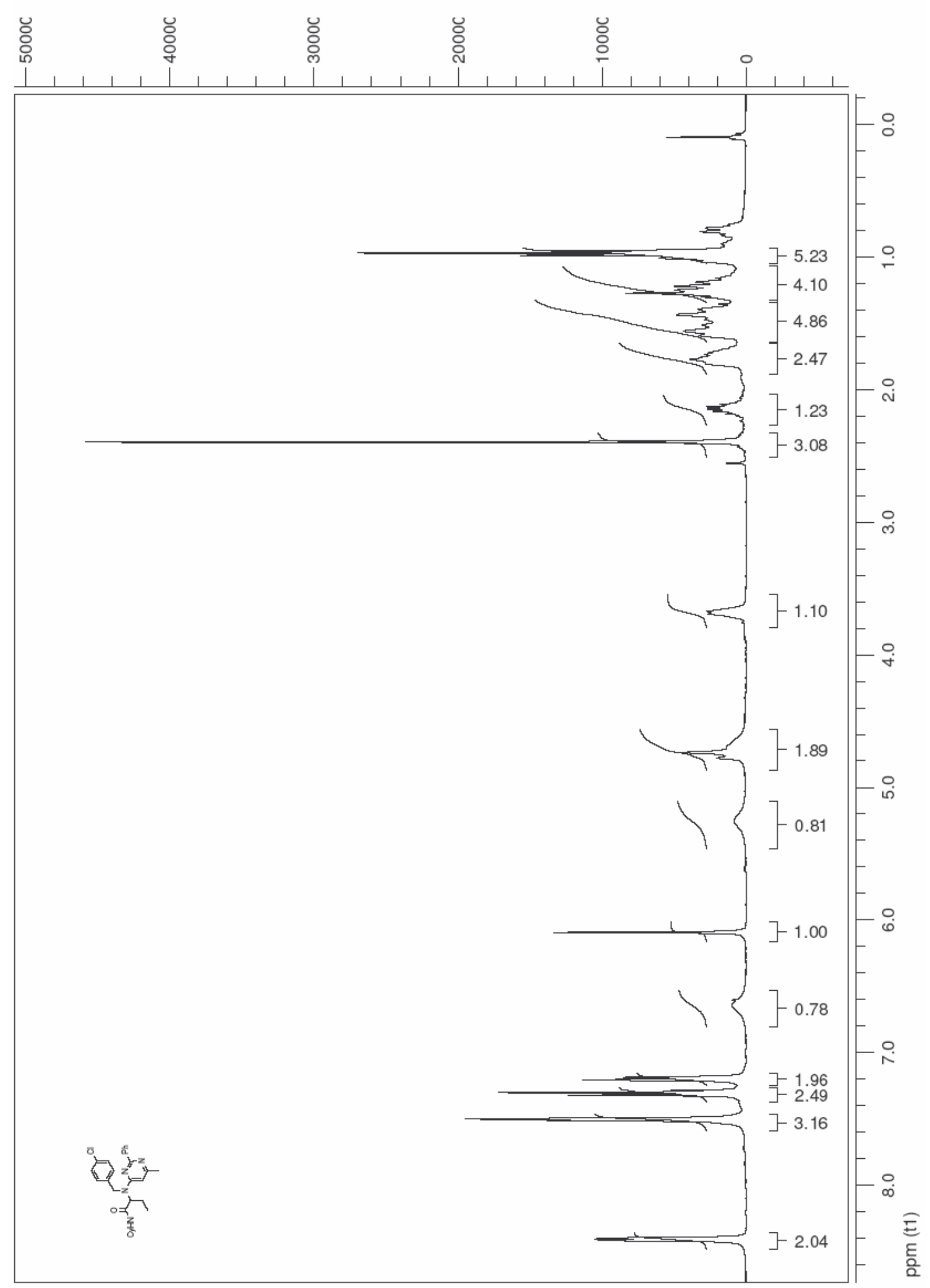

Table 1, Entry 8. 


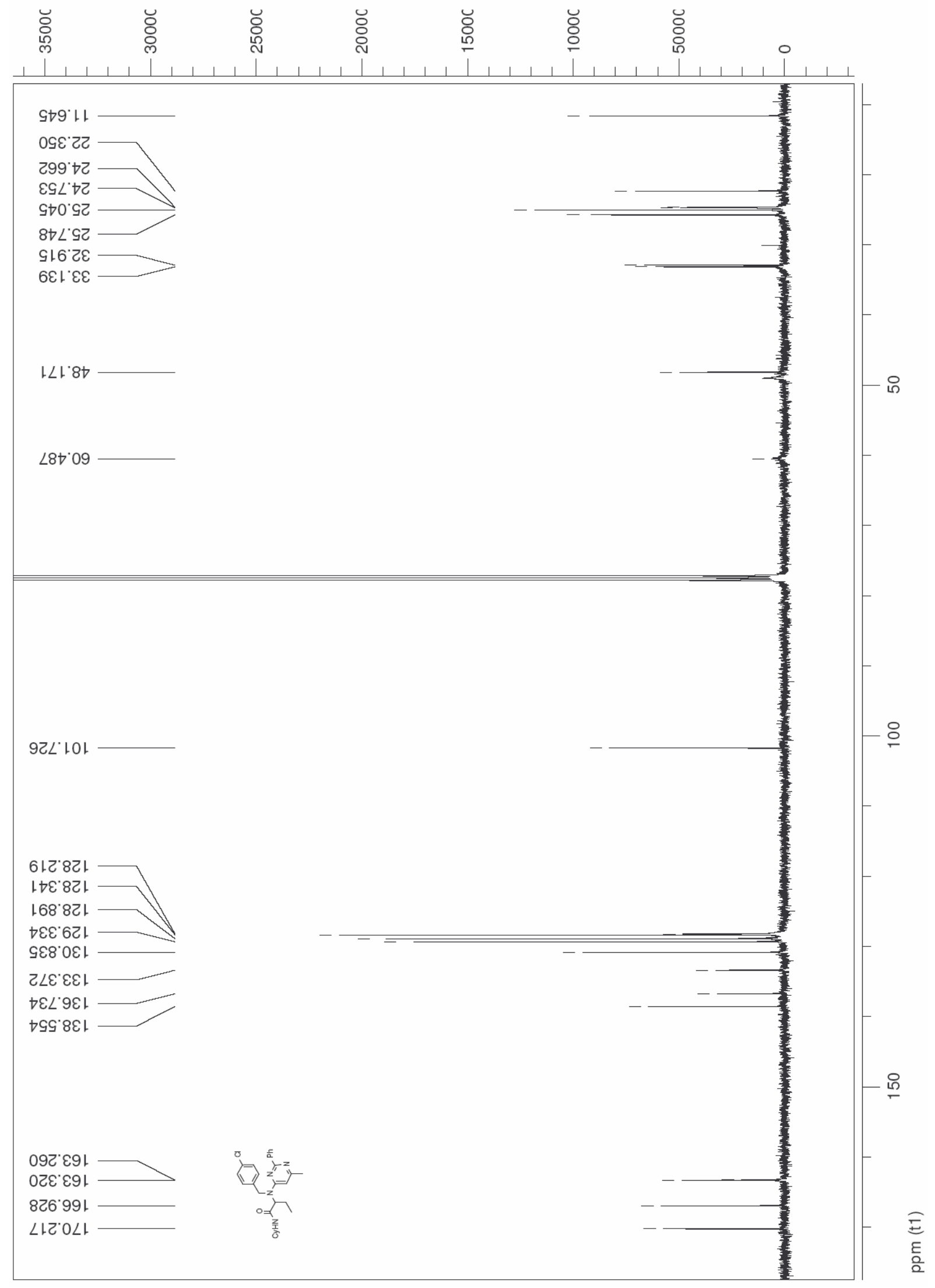


Table 1, Entry 8. 


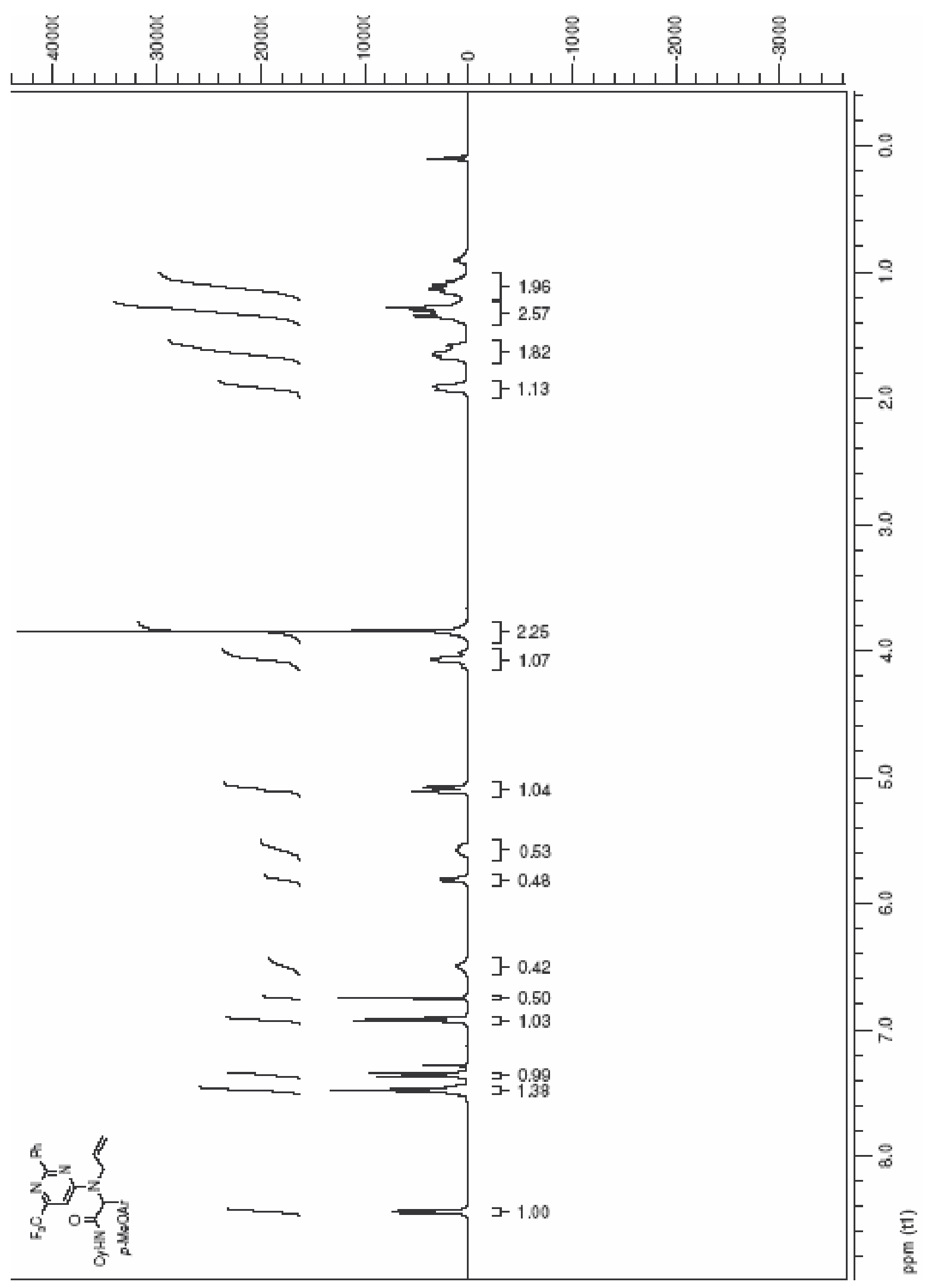

Table 1, Entry 9. 


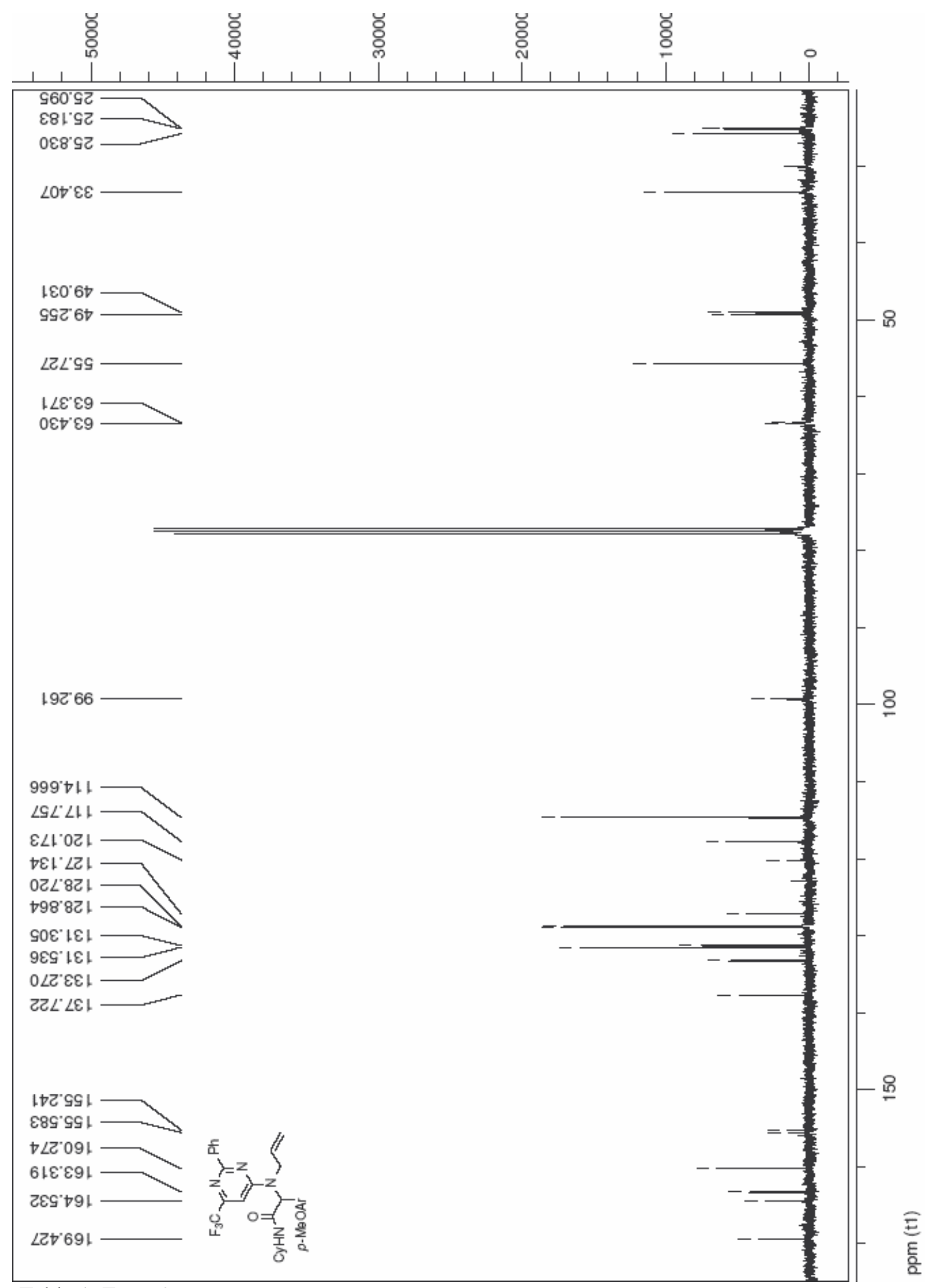

Table 1, Entry 9. 


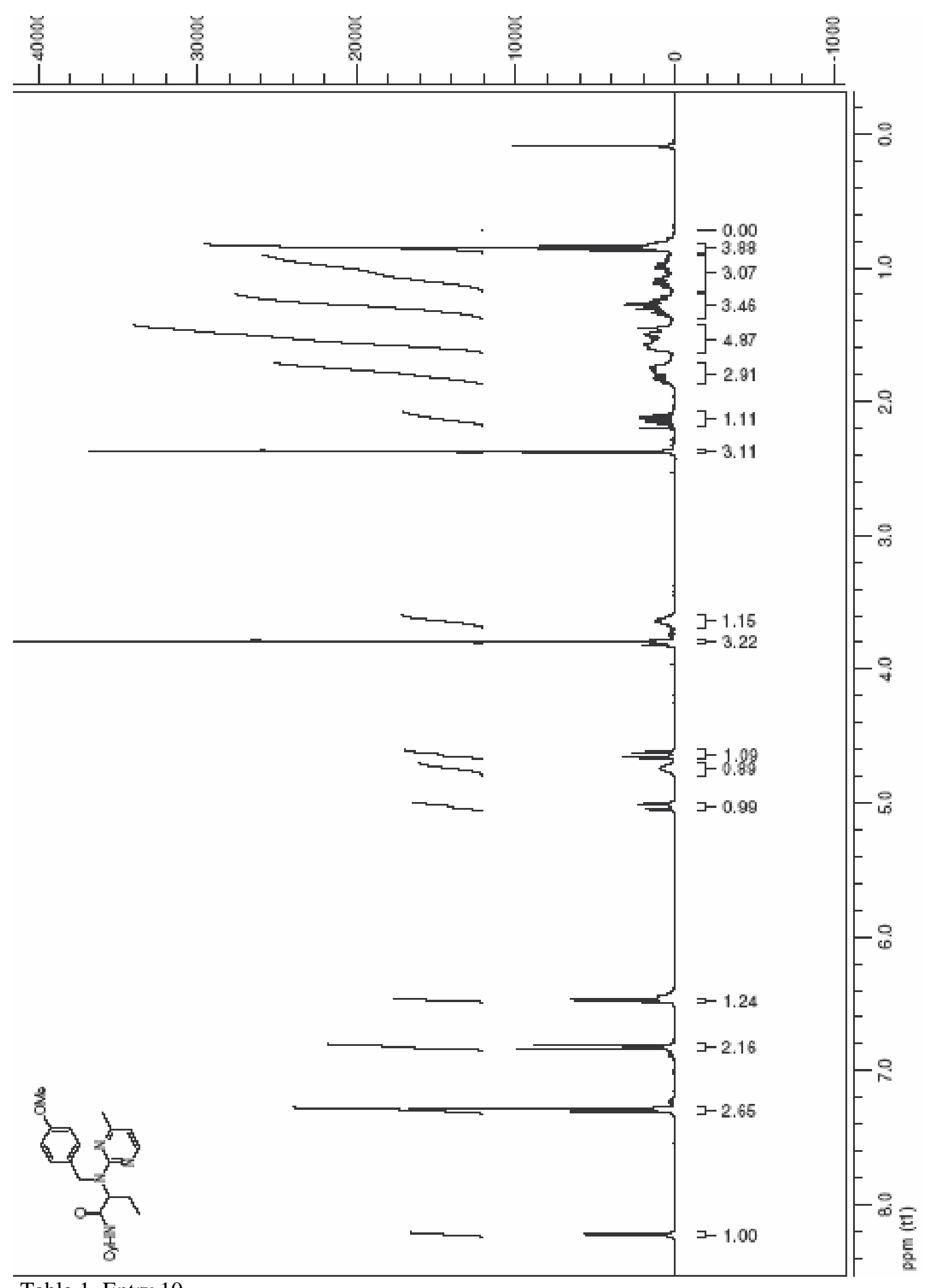

Table 1, Entry 10. 


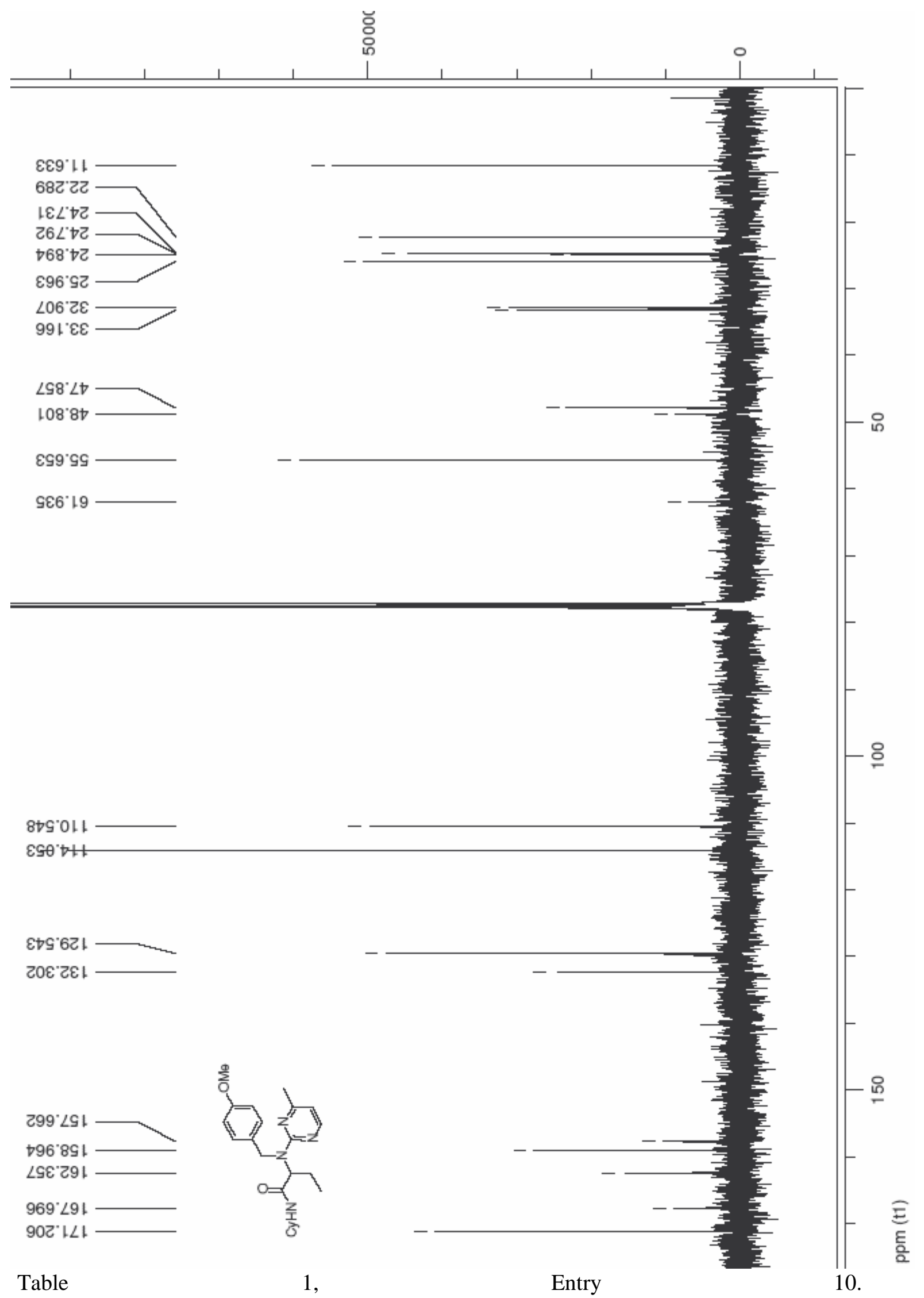




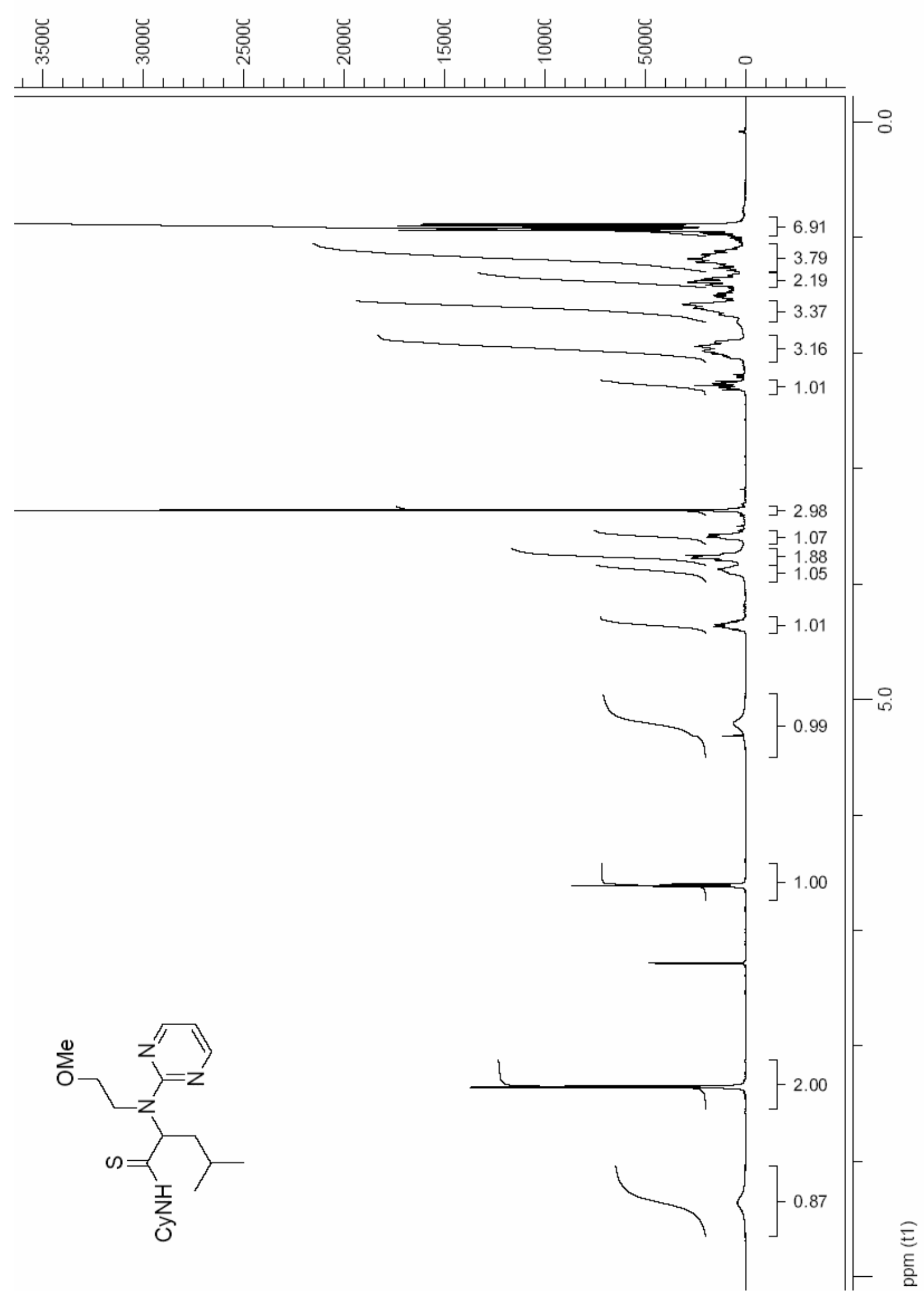


Table 2, Entry 1. 


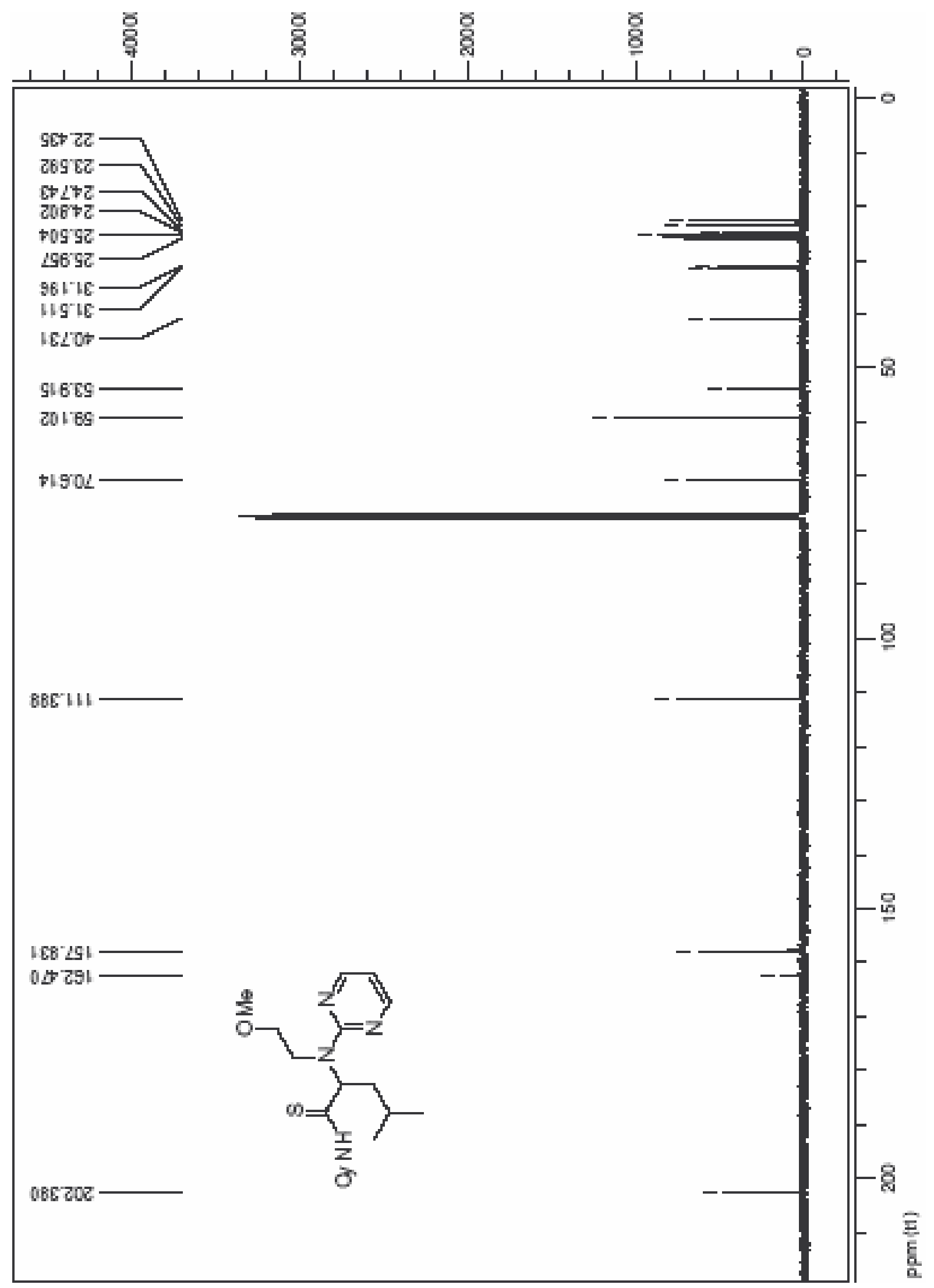

Table 2, Entry 1. 


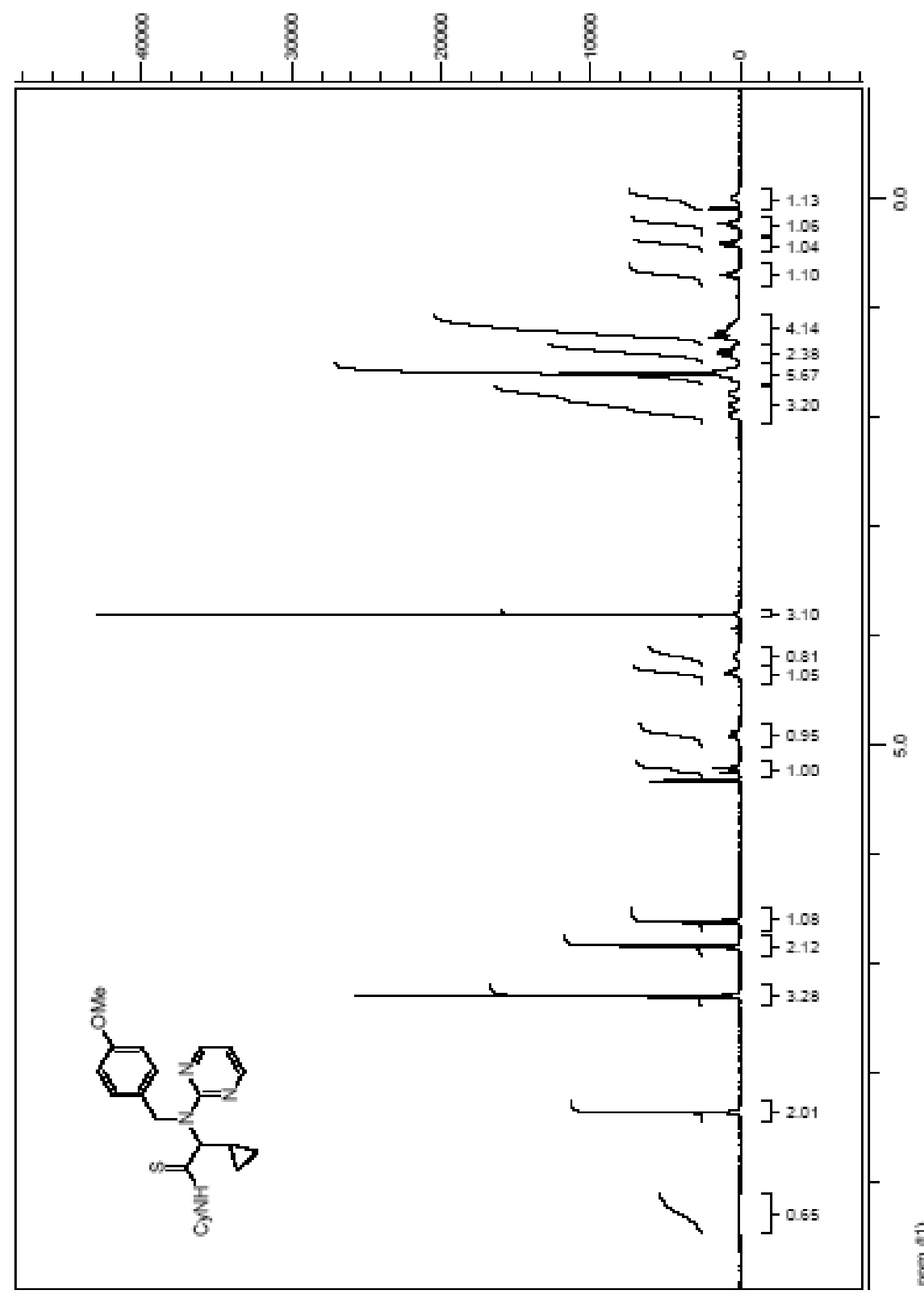


Table 2, Entry 2. 


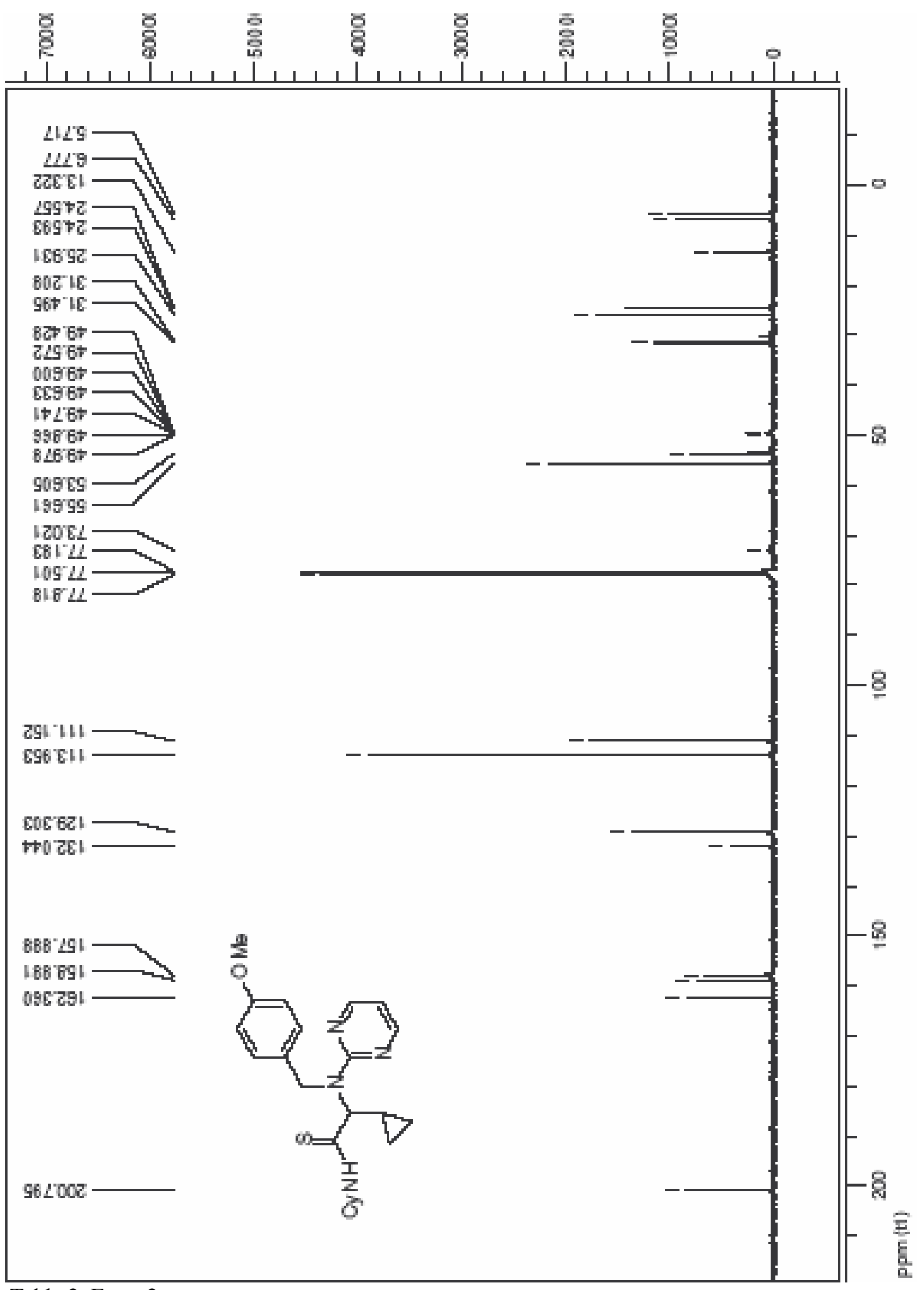

Table 2, Entry 2. 


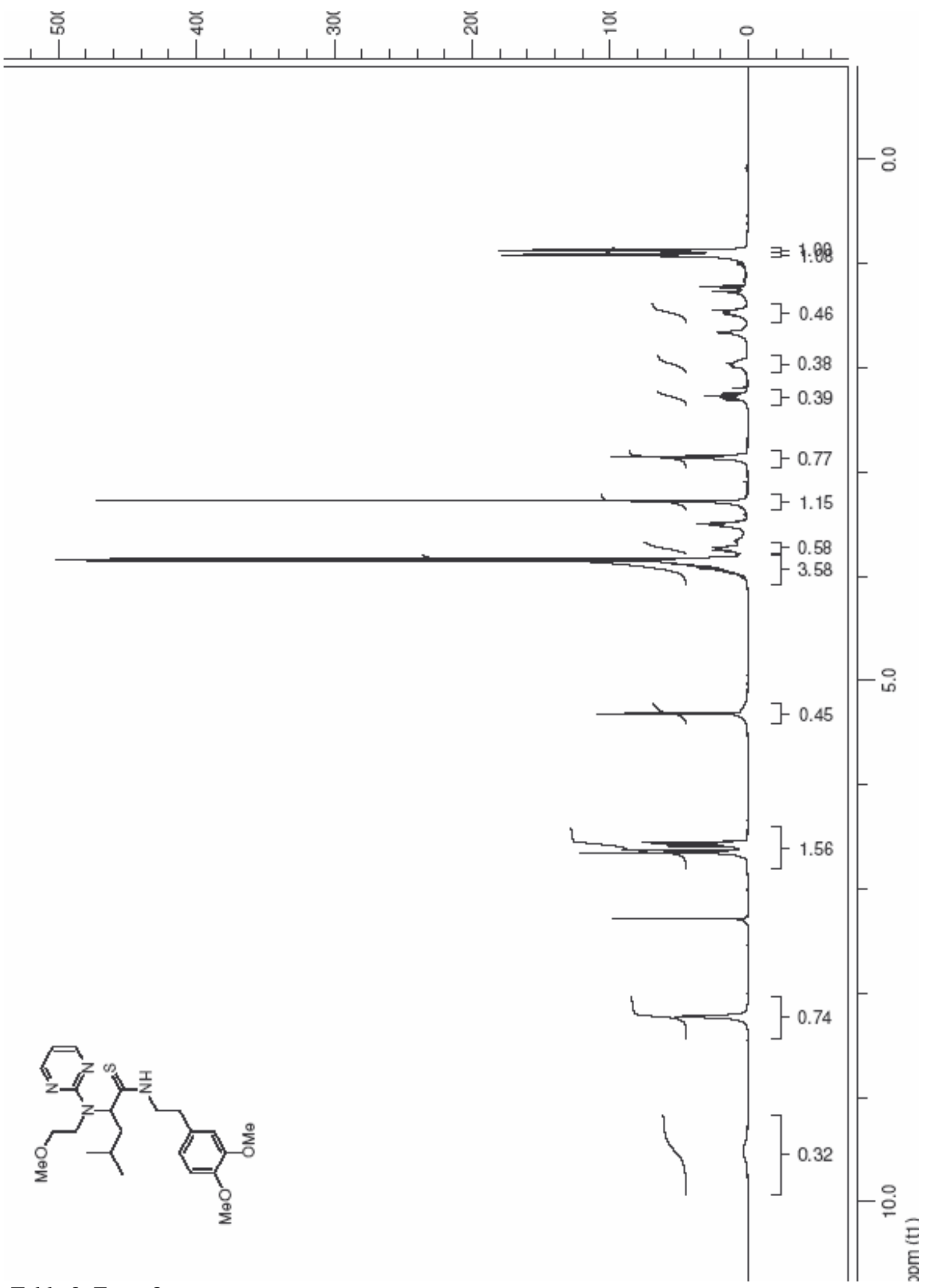

Table 2, Entry 3. 


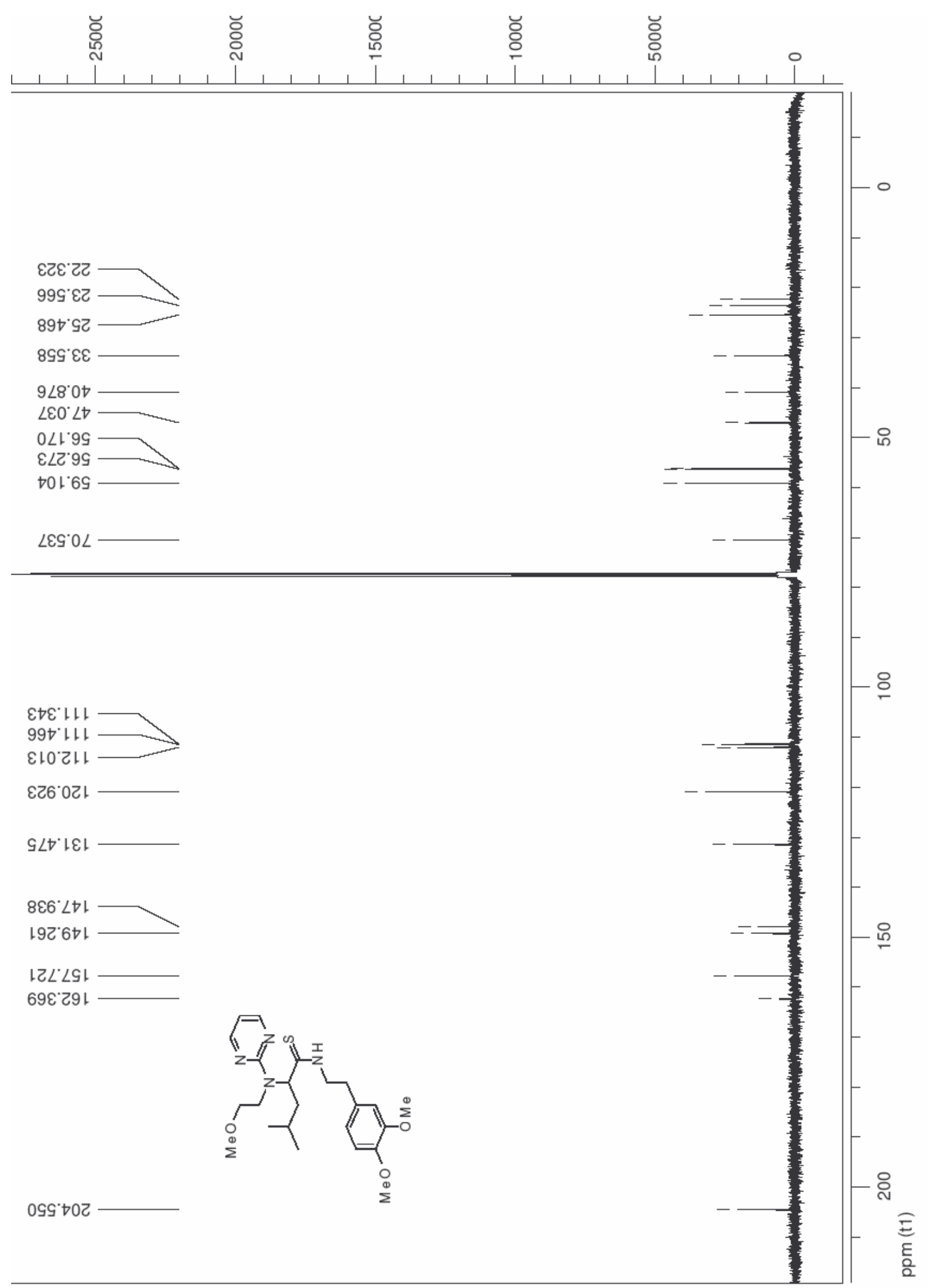

Table 2, Entry 3. 


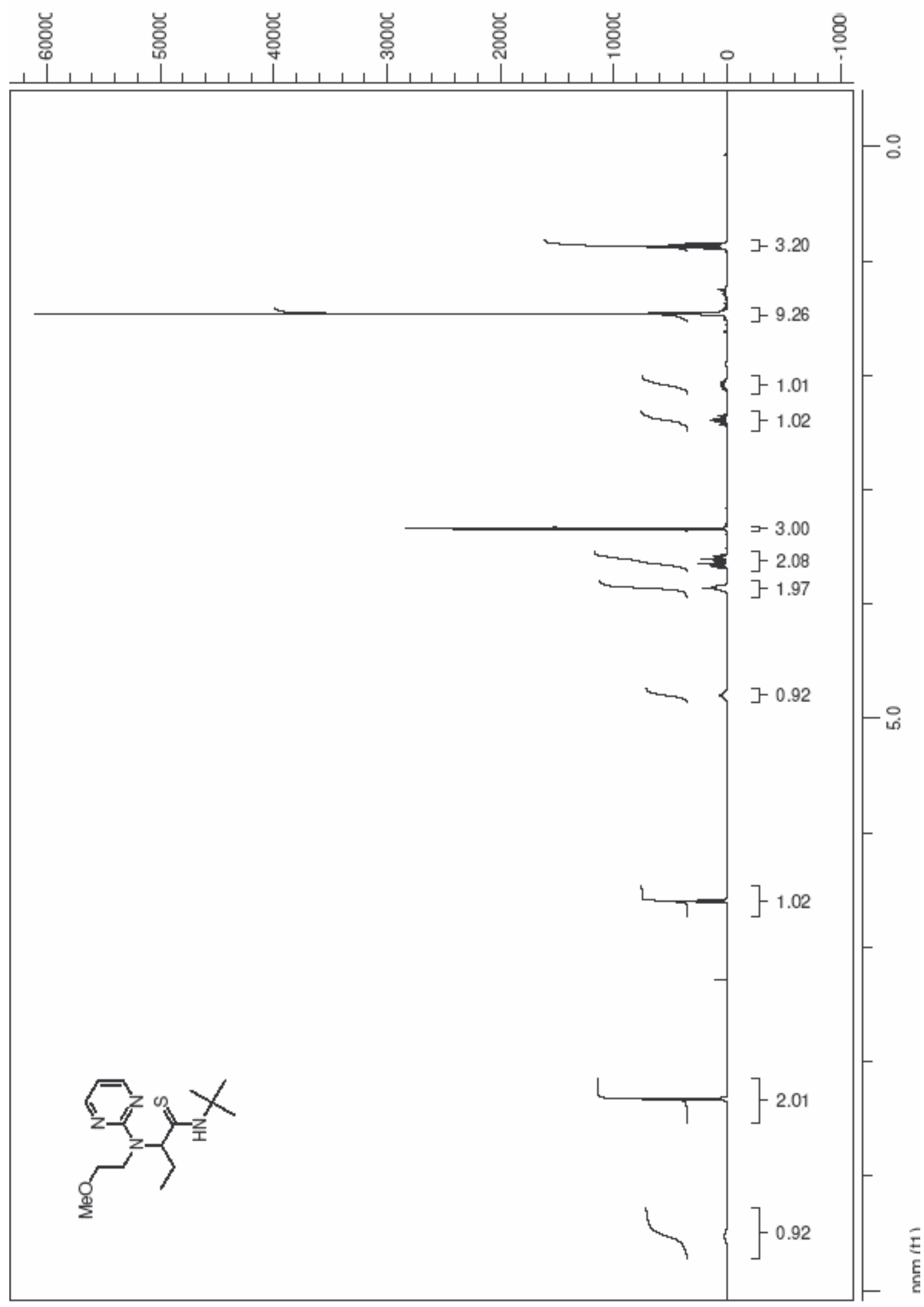

Table 2, Entry 4. 


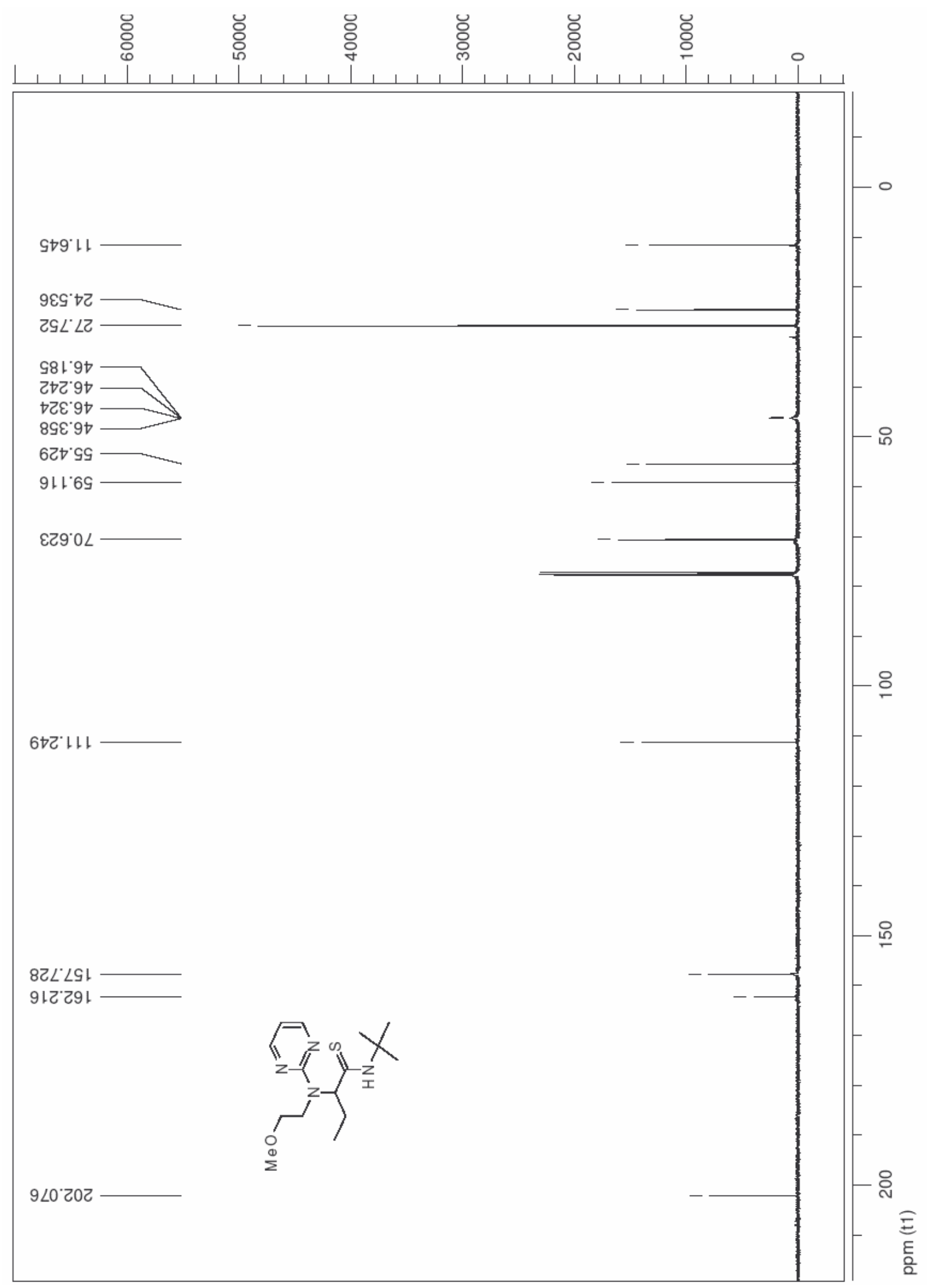

Table 2, Entry 4. 